\title{
High order WENO and DG methods for time-dependent convection-dominated PDEs: a brief survey of several recent developments ${ }^{1}$
}

\author{
Chi-Wang Shu \\ Division of Applied Mathematics \\ Brown University \\ Providence, RI 02912, USA \\ E-mail: shu@dam.brown.edu
}

\begin{abstract}
For solving time-dependent convection-dominated partial differential equations (PDEs), which arise frequently in computational physics, high order numerical methods, including finite difference, finite volume, finite element and spectral methods, have been undergoing rapid developments over the past decades. In this article we give a brief survey of two selected classes of high order methods, namely the weighted essentially non-oscillatory (WENO) finite difference and finite volume schemes and discontinuous Galerkin (DG) finite element methods, emphasizing several of their recent developments: bound-preserving limiters for DG, finite volume and finite difference schemes, which address issues in robustness and accuracy; WENO limiters for DG methods, which address issues in non-oscillatory performance when there are strong shocks, and inverse Lax-Wendroff type boundary treatments for finite difference schemes, which address issues in solving complex geometry problems using Cartesian meshes.
\end{abstract}

Key Words: high order schemes; time-dependent convection-dominated partial differential equations; finite difference schemes; finite volume schemes; discontinuous Galerkin method; bound-preserving limiters; WENO limiters; inverse Lax-Wendroff boundary treatments.

\footnotetext{
${ }^{1}$ Research supported by AFOSR grant F49550-12-1-0399, ARO grant W911NF-15-1-0226, DOE grant DE-FG02-08ER25863 and NSF grant DMS-1418750.
} 


\section{Introduction}

High order numerical methods are attractive in computational physics because of their potential in achieving desired resolution with a small number of degrees of freedom, which often leads to savings in computational cost. Another advantage of high order schemes is that, on machines with a fixed capacity (memory), using high order methods allows better achievable resolution and accuracy. This is why the benchmark results in direct numerical simulation of turbulent flows are often obtained by spectral methods, high order compact schemes, or other high order methods.

This article is mainly concerned with the numerical solution of time-dependent convectiondominated partial differential equations (PDEs), which arise frequently in computational physics. Examples include high Reynolds number Navier-Stokes equations, Maxwell equations and other linear or nonlinear wave equations, magneto-hydrodynamics (MHD), rela-

tivistic hydrodynamics (RHD), etc. For such problems, solutions often contain sharp gradient regions or discontinuities, making their numerical simulation more challenging, especially for high order schemes. Nevertheless, because of their good potential in efficiency, high order methods for solving time-dependent convection-dominated PDEs have been actively pursued in the literature in the last decades. Many types of numerical methods have been investigated. On structured meshes over regular geometry, finite difference schemes (including compact schemes) and spectral methods are most popular, because of their simplicity and cost-effectiveness. On unstructured meshes over complex geometry, finite volume schemes and finite element methods (including discontinuous Galerkin methods) are more popular, because of their flexibility on arbitrary meshes. Other types of methods such as meshless methods over arbitrary point clouds have also been designed.

This article gives a brief survey of two classes of high order methods for solving timedependent convection-dominated PDEs, namely the finite difference and finite volume weighted essentially non-oscillatory (WENO) schemes, and the discontinuous Galerkin (DG) methods, emphasizing a few of their recent developments. We are not attempting to be comprehensive 
in this survey and do not cover all available classes of high order methods, and within the two classes of high order methods which we do cover, we do not attempt to discuss all of their recent developments. Rather, we concentrate only on a few selected cases of recent developments of these methods, in order to highlight their utility and values. We will concentrate on the spatial discretizations, and will use mostly the explicit strong-stability-preserving (SSP) Runge-Kutta or multi-step methods [203, 207, 82, 81] as examples of time discretization.

The structure of this article is as follows. In section 2 we describe two classes of high order methods for solving time-dependent convection-dominated PDEs, namely the finite difference and finite volume WENO schemes, and DG and related methods. In sections 3 to 5 , we discuss three different topics of recent developments, including bound-preserving limiters, WENO limiters for DG schemes, and inverse Lax-Wendroff type boundary conditions for finite difference schemes, respectively. Concluding remarks are given in section 6 .

\section{Two classes of high order methods}

In this section we give a brief discussion of two classes of high order methods for solving time-dependent convection-dominated PDEs, namely the finite difference and finite volume WENO schemes, and DG and related methods.

\subsection{Finite difference and finite volume WENO schemes}

A finite difference scheme approximates the point values of the exact solution on a given grid. Let us use the one-dimensional conservation law

$$
u_{t}+f(u)_{x}=0
$$

to show the idea. The finite difference solution $u_{j}$ approximates the exact solution $u\left(x_{j}, t\right)$ at the grid point $x_{j}$, in a semi-discrete (time continuous) version. To effectively compute weak solutions (which may contain discontinuities) of the conservation law (2.1), we would use conservative difference to approximate the derivative $f(u)_{x}$ at the grid point $x_{j}$ :

$$
\frac{d}{d t} u_{j}+\frac{1}{\Delta x}\left(\hat{f}_{j+1 / 2}-\hat{f}_{j-1 / 2}\right)=0
$$


where $\Delta x$ is the spatial mesh size (assumed to be uniform here for simplicity), and $\hat{f}_{j+1 / 2}$ is the numerical flux, which typically is a Lipschitz continuous function of several neighboring values $u_{i}$. In [208], a general procedure to form the numerical flux based on the point values of the solution to guarantee accuracy on uniform meshes is described, which provides formulas for all high order conservative finite difference schemes and have been used widely.

The WENO schemes $[153,120]$ were based on the earlier work of essentially non-oscillatory (ENO) schemes $[93,207]$. Let us use the simple case with $f^{\prime}(u) \geq 0$ and fifth order finite difference WENO scheme in [120] as an example to demonstrate the basic idea. The fifth order numerical flux is given by

$$
\hat{f}_{j+1 / 2}=w_{1} \hat{f}_{j+1 / 2}^{(1)}+w_{2} \hat{f}_{j+1 / 2}^{(2)}+w_{3} \hat{f}_{j+1 / 2}^{(3)}
$$

where $\hat{f}_{j+1 / 2}^{(i)}$, for $i=1,2,3$, are three third order fluxes on three different small stencils given by

$$
\begin{aligned}
& \hat{f}_{j+1 / 2}^{(1)}=\frac{1}{3} f\left(u_{j-2}\right)-\frac{7}{6} f\left(u_{j-1}\right)+\frac{11}{6} f\left(u_{j}\right), \\
& \hat{f}_{j+1 / 2}^{(2)}=-\frac{1}{6} f\left(u_{j-1}\right)+\frac{5}{6} f\left(u_{j}\right)+\frac{1}{3} f\left(u_{j+1}\right), \\
& \hat{f}_{j+1 / 2}^{(3)}=\frac{1}{3} f\left(u_{j}\right)+\frac{5}{6} f\left(u_{j+1}\right)-\frac{1}{6} f\left(u_{j+2}\right),
\end{aligned}
$$

and the nonlinear weights $w_{i}$ are given by

$$
w_{i}=\frac{\tilde{w}_{i}}{\sum_{k=1}^{3} \tilde{w}_{k}}, \quad \tilde{w}_{k}=\frac{\gamma_{k}}{\left(\varepsilon+\beta_{k}\right)^{2}},
$$

with the linear weights $\gamma_{k}$ given by

$$
\gamma_{1}=\frac{1}{10}, \quad \gamma_{2}=\frac{3}{5}, \quad \gamma_{3}=\frac{3}{10}
$$

and the smoothness indicators $\beta_{k}$ given by

$$
\begin{aligned}
& \beta_{1}=\frac{13}{12}\left(f\left(u_{j-2}\right)-2 f\left(u_{j-1}\right)+f\left(u_{j}\right)\right)^{2}+\frac{1}{4}\left(f\left(u_{j-2}\right)-4 f\left(u_{j-1}\right)+3 f\left(u_{j}\right)\right)^{2} \\
& \beta_{2}=\frac{13}{12}\left(f\left(u_{j-1}\right)-2 f\left(u_{j}\right)+f\left(u_{j+1}\right)\right)^{2}+\frac{1}{4}\left(f\left(u_{j-1}\right)-f\left(u_{j+1}\right)\right)^{2} \\
& \beta_{3}=\frac{13}{12}\left(f\left(u_{j}\right)-2 f\left(u_{j+1}\right)+f\left(u_{j+2}\right)\right)^{2}+\frac{1}{4}\left(3 f\left(u_{j}\right)-4 f\left(u_{j+1}\right)+f\left(u_{j+2}\right)\right)^{2} .
\end{aligned}
$$


Finally, $\varepsilon$ is a parameter to avoid the denominator to become 0 and is usually taken as $\varepsilon=10^{-6}$ in the computation. This construction of WENO fluxes can be generalized to the case of $f^{\prime}(u)$ changing sign (using flux splitting such as the Lax-Friedrichs flux splitting), to the case of one-dimensional systems (using local characteristic decomposition), and to the case of two and more spatial dimensions (fluxes for each coordinate direction, corresponding to $f(u)_{x}, g(u)_{y}$, etc., are computed using the one-dimensional formulae (2.2)-(2.7) with grid points in that direction only, namely in a dimension-by-dimension fashion), we refer to, e.g., $[120,204]$ for more details. WENO schemes are available for arbitrarily high order accuracy [8]. The review paper [205] summarizes the development and application of WENO schemes until 2006.

Even though most of the finite difference WENO schemes in applications use the numerical flux construction in (2.2)-(2.7), which reconstructs directly from the physical fluxes $f\left(u_{i}\right)$, the earlier paper [207] actually provided an alternative formulation of the numerical flux, which could rely on the WENO interpolation directly on the point values $u_{i}$. This alternative approach has been explored more recently in $[122,123]$. It is slightly more expensive than the one in $[208,120]$, as outlined in (2.2)-(2.7) above, but it has a few distinct advantages, including its flexibility to use arbitrary monotone fluxes or approximate Riemann solvers [122], its narrower effective stencil with a Lax-Wendroff time discretization [122], and its ability to preserve free-stream solution exactly on curvilinear time-dependent meshes [123]. It is well-known that free-stream preserving is difficult for finite difference schemes on curvilinear time-dependent meshes, see, e.g. [220, 269, 231, 171, 211] for discussions on this issue. The approach in [123] is one of the very few high order finite difference schemes which can maintain exactly free-stream solutions on time-dependent meshes when the nonlinear WENO (or other nonlinear limiting) procedure is used in the spatial discretization.

A particular class of finite difference schemes, for which the numerical flux $\hat{f}_{j+1 / 2}$ relies on all grid points via a banded matrix inversion, is called compact schemes [139]. For the same order of accuracy, compact schemes usually give much smaller dispersion and/or 
dissipation errors, hence they are more suitable for problems where marginally resolved waves are important, such as turbulence simulations. The WENO idea can be combined with compact schemes, resulting in weighted compact schemes which can improve robustness for shocked flows, e.g. in $[54,121,179,191,271,154,213]$.

In one space dimension, finite volume schemes are very similar to finite difference schemes. The major difference is that a finite volume scheme approximates the cell averages $\bar{u}\left(x_{j}, t\right)=$ $\frac{1}{\Delta x_{j}} \int_{x_{j-1 / 2}}^{x_{j+1 / 2}} u(x, t) d x$ of the exact solution on a given mesh. For the one-dimensional conservation law (2.1), the finite volume solution $\bar{u}_{j}$ approximates the cell average of the exact solution $\bar{u}\left(x_{j}, t\right)$ in the cell $I_{j}=\left(x_{j-1 / 2}, x_{j+1 / 2}\right)$. The scheme is still conservative

$$
\frac{d}{d t} \bar{u}_{j}+\frac{1}{\Delta x_{j}}\left(\hat{f}_{j+1 / 2}-\hat{f}_{j-1 / 2}\right)=0
$$

where the numerical flux $\hat{f}_{j+1 / 2}$ now depends on several neighboring cell averages $\bar{u}_{i}$. The computation of the numerical flux $\hat{f}_{j+1 / 2}$ for a finite volume scheme depends on a monotone flux or an exact or approximate Riemann solver, namely $\hat{f}_{j+1 / 2}=\hat{f}\left(u_{j+1 / 2}^{-}, u_{j+1 / 2}^{+}\right)$, where $u_{j+1 / 2}^{ \pm}$are reconstructed from the cell averages $\bar{u}_{i}$ nearby. The reconstruction procedure from $\bar{u}_{i}$ to $u_{j+1 / 2}^{-}$is completely the same as $(2.2)-(2.7)$ in finite difference, except that $f\left(u_{i}\right)$ is now replaced by $\bar{u}_{i}$ and the final product $\hat{f}_{j+1 / 2}$ is replaced by $u_{j+1 / 2}^{-}$. The construction of $u_{j+1 / 2}^{+}$is mirror symmetric to that for $u_{j+1 / 2}^{-}$with respect to $x_{j+1 / 2}$. Therefore, in one space dimension, the codes and computational costs for finite difference and finite volume WENO schemes are almost identical, and they produce similar computational results. However, in multidimensions, finite volume schemes are much more costly than finite difference schemes, since a reconstruction from multi-dimensional cell averages to point values needs to be performed and numerical quadratures must be used for the integration to get numerical fluxes. We refer to $[204,25,270]$ for the discussion of multi-dimensional finite volume schemes and their comparison with the finite difference counter-parts. However, finite volume schemes have a significant advantage over finite difference schemes, in that they can be designed on nonsmooth and unstructured meshes without losing their high order accuracy and conservation properties, see, for example, [1, 72, 103, 66, 283] for high order finite volume ENO and 
WENO schemes on two- and three-dimensional unstructured meshes.

Efforts have been made in the literature to improve the accuracy, dispersion or dissipation quality, or the efficiency of WENO schemes, via modifying the linear or nonlinear weights, via modifying the smoothness indicators, or via hybridizing WENO with other types of schemes. For example, an improved WENO scheme (in terms of accuracy and resolution) is provided in [18], which is further developed in [26] as the WENO-Z scheme. Accuracy issues regarding WENO schemes are discussed and improvements made in [96, 57]. Efforts to improve the dissipation and / or dispersion properties of WENO schemes for high frequency wave computations are made in $[241,180,161,107,118,260]$.

One research direction regarding WENO schemes which has attracted a lot of attention recently is adaptivity, aiming at reducing computational cost to achieve desired resolution at places where it is needed. For example, an adaptive mesh refinement (AMR) WENO finite difference scheme for multi-scale simulations is developed in [200] and an AMR WENO finite difference solver for multi-dimensional detonation is developed in [234]. A waveletbased adaptive WENO algorithm is constructed for Euler equations in [144]. A space-time adaptive ADER-WENO finite volume scheme is constructed in [65], and an adaptive mesh WENO method is developed in [104].

Applications of WENO schemes can be found in many areas of computational physics and computational engineering. We list below only a few examples over the past year (since 2015). Recent applications of WENO schemes can be found in the simulations of astrophysics and geophysics [55, 83, 137, 140, 162, 172, 215, 223], atmospheric and climate science [70, 181, 225], batch chromatographic separation [101], biomolecular solvation [286], bubble clusters in fluids [222], combustion [11, 15, 24, 164, 214, 268], detonation waves [92, 114, 145, 233], elastic-plastic solids [173], flame structure [261], granular gas [3], hypersonic flows [109], infectious disease models [209], laser welding [174], magnetohydrodynamics [20, 166], mathematical finance for solving the Black-Scholes equation [90], multiphase and multispecies flows $[13,91,108,110,159,160,239]$, networks and blood flows [168], ocean 
waves [28, 125], oil storage process [196], rarefied gas flow [147], rotor aerodynamic performance [117], semiconductor device and other computational electronics [59, 80, 165], shallow water equations [29, 128, 129, 138], special relativistic hydrodynamics [244, 264], supersonic flows [267, 280], and turbulent flows [74, 75, 131, 150, 193, 258]. This very incomplete list over just one year period clearly demonstrates the wide-spread influence of the WENO technique in computational science and engineering.

\subsection{Discontinuous Galerkin and related schemes}

Similar to a finite volume scheme, a discontinuous Galerkin (DG) method for a conservation law such as (2.1) also approximates an integral version of it. Instead of integrating the PDE (2.1) over the interval $I_{j}=\left(x_{j-1 / 2}, x_{j+1 / 2}\right)$ directly, we first multiply it by a test function $v(x)$, then integrate over the interval $I_{j}$ and integrate by parts to obtain

$\int_{I_{j}} u_{t}(x, t) v(x) d x-\int_{I_{j}} f(u(x, t)) v_{x}(x) d x+f\left(u\left(x_{j+1 / 2}, t\right)\right) v\left(x_{j+1 / 2}\right)-f\left(u\left(x_{j-1 / 2}, t\right)\right) v\left(x_{j-1 / 2}\right)=0$.

The numerical solution for a DG scheme is represented by a piecewise polynomial function, still denoted as $u(x, t)$, which is a polynomial of degree $k$ in each cell $I_{j}$. The test function $v(x)$ is also taken as a piecewise polynomial of degree $k$. If we would like to convert (2.8) into a scheme, we would need to interpret $f\left(u\left(x_{j \pm 1 / 2}, t\right)\right)$ and $v\left(x_{j \pm 1 / 2}\right)$ suitably, as both the solution $u(x, t)$ and the test function $v(x)$ are discontinuous at the interface $x_{j \pm 1 / 2}$. Based on the similarity with finite volume schemes, we can take the piecewise constant case and attempt to recover the traditional first order monotone scheme. This will determine the choices of the interface terms: $f\left(u\left(x_{j \pm 1 / 2}, t\right)\right)$ should be replaced by a numerical flux $\hat{f}\left(u\left(x_{j \pm 1 / 2}^{-}, t\right), u\left(x_{j \pm 1 / 2}^{+}, t\right)\right)$, and $v\left(x_{j \pm 1 / 2}\right)$ should be replaced by its traces inside the cell $I_{j}$, namely by $v\left(x_{j+1 / 2}^{-}\right)$at $x_{j+1 / 2}$ and by by $v\left(x_{j-1 / 2}^{+}\right)$at $x_{j-1 / 2}$. The DG scheme is thus obtained: we would like to find a piecewise polynomial of degree $k$, still denoted by $u(x, t)$, such that the following equality holds for all test functions $v(x)$ which are piecewise polynomials of 
degree $k$ :

$$
\begin{array}{r}
\int_{I_{j}} u_{t}(x, t) v(x) d x-\int_{I_{j}} f(u(x, t)) v_{x}(x) d x+\hat{f}\left(u\left(x_{j+1 / 2}^{-}, t\right), u\left(x_{j+1 / 2}^{+}, t\right)\right) v\left(x_{j+1 / 2}^{-}\right) \\
-\hat{f}\left(u\left(x_{j-1 / 2}^{-}, t\right), u\left(x_{j-1 / 2}^{+}, t\right)\right) v\left(x_{j-1 / 2}^{+}\right)=0 .
\end{array}
$$

Similar to finite volume schemes, the DG method (2.9) can also be evolved explicitly by any ODE solver, without the need to solve any global system. The generalization of the DG method to multi-dimensional unstructured meshes is straightforward, as there is no reconstruction needed as in finite volume schemes. On the same mesh, in comparison with finite volume schemes of the same order of accuracy, the DG method saves in the computational cost of reconstruction, but has a much larger memory requirement and also higher cost in evolving all these extra degrees of freedom in time. On balance, whether a finite volume scheme or a DG scheme is more cost-effective to reach the same level of errors depends on the specific PDE, the complexity of the geometry and meshes, and the computer platform. A comparison of the relative efficiency between the finite volume and DG schemes is given in [287]. There are also intermediate methods between DG and finite volume schemes, which have more than one degrees of freedom per cell yet not enough for the full $k$-th degree polynomial, hence still require a reconstruction which however has a smaller stencil than that for regular DG schemes, such as the Hermite-type finite volume and finite difference schemes $[184,185,151]$, the recovery-type DG schemes $[226,227]$, and the $P^{n} P^{m}$ type methods $[63,62]$. If the high memory requirement of DG is a concern, these intermediate methods might be good options. We would like to mention that the DG method is extremely local in data communications. The evolution of the solution in each cell needs to communicate only with its immediate neighbors, regardless of the order of accuracy. The methods thus have excellent parallel efficiency, usually more than $99 \%$ for a fixed mesh, and more than $80 \%$ for a dynamic load balancing with adaptive meshes which change often during time evolution, see, e.g. $[16,189,4,12]$. The DG method is also very friendly to the GPU environment [130]. 
The first DG method was introduced in 1973 by Reed and Hill [188], in the framework of neutron transport, i.e. a time independent linear hyperbolic equation. It was later developed for solving nonlinear hyperbolic conservation laws by Cockburn et al. in a series of papers $[44,43,40,45]$, in which the authors have established a framework to easily solve nonlinear time dependent problems, such as the Euler equations in compressible gas dynamics, using explicit, nonlinearly stable high order Runge-Kutta time discretizations [207] and DG discretization in space described above. Generalizations to convection-diffusion equations were carried out in, e.g. $[9,46,10]$, and to PDEs with higher order derivatives in, e.g. $[254,255,251,33]$.

For the history and development of the DG method, we refer to the survey paper [41], and other papers in that Springer volume, which contains the conference proceedings of the First International Symposium on Discontinuous Galerkin Methods held at Newport, Rhode Island in 1999. The lecture notes [39] is a good reference for many details, as well as the extensive review paper [47]. The review paper [252] covers the local DG method for partial differential equations (PDEs) containing higher order spatial derivatives, such as Navier-Stokes equations. There are three special issues devoted to the discontinuous Galerkin methods [48, 50, 49], which contain many interesting papers in the development of the method in all aspects including algorithm design, analysis, implementation and applications. There are also a few recent books and lecture notes $[56,100,126,141,194,206]$ on DG methods.

One advantage of the DG framework is that it can easily accommodate local approximation spaces, which do not have to be polynomials, with special properties, such as locally divergence-free or curl-free elements, or spaces fitting particular properties of the PDE solutions. This flexibility can be explored to design structure-preserving DG methods which can better approximate the relevant PDE with less computational cost. See for example the locally divergence-free DG method for the Maxwell equations [42] and for the MHD equations [142], and the locally curl-free DG method for the Hamilton-Jacobi equations [143] and for 
the multiscale modeling of dynamics of crystalline solids [237]. Exponential or trigonometric local spaces have been studied in $[262,288]$, plain wave local spaces are used to solve the Helmholtz equation in $[27,115,76]$, and suitable multiscale basis functions obtained from asymptotic analysis are used for multiscale problems in [263, 238, 236, 69, 281, 58].

Another advantage of the DG framework is its easiness to accommodate arbitrary $h-p$ adaptivity. Of course, one would need suitable error indicators to guide local decisions on whether to refine or coarsen the mesh, or to increase or decrease the polynomial degree. Various superconvergence results and a posteriori error estimates are pursued towards this goal, see for example [5, 6, 22, 23, 34, 35, 105, 127, 163, 256].

There are a few classes of numerical methods studied in the literature which are closely related to discontinuous Galerkin methods. Examples include the spectral finite volume (SV) method [240], spectral finite difference (SD) method [156], staggered-grid (SG) multidomain spectral method [132], and correction procedure via reconstruction (CPR) method [116, 242, 89]. Many of these methods can be considered as discontinuous Galerkin methods with suitable and clever choices of numerical integration quadratures, and they often reduce to discontinuous Galerkin methods for linear problems with suitable choices of parameters. These methods may provide certain conceptual simplicity over DG methods, for example the CPR methods can be defined on solution points and hence they look like finite difference methods, similar to the so-called nodal DG methods [100] which uses numerical quadrature and solution values at quadrature points as degrees of freedom.

Applications of DG methods can be found in many areas of computational physics and computational engineering. We list below only a few examples over the past year (since 2015). Recent applications of DG methods can be found in the simulations of the CahnHilliard-Brinkman system [85], compressible flow in the transonic axial compressor [190], computational astrophysics [197], computational geosciences [221], elastodynamics [53], flow instabilities [51], Fokker-Planck equations [152], fractional PDEs [111, 243], front propagation with obstacles [17], functionalized Cahn-Hilliard equation [87], interfaces [278], magnetohy- 
drodynamics [265], moment closures for kinetic equations [2], multi-phase flow and phase transition [52, 169], Navier-Stokes and Boussinesq equations [64, 224], nonlinear Schrodinger equation [86, 149, 158], ocean waves [192], population models [112], porous media [84], rarefied gas [212], semiconductor device simulation [155], shallow water equations [73], thin film epitaxy problem [247], traffic flow and networks [21], three-dimensional flows [175], turbulent flows [246], underwater explosion [235], viscous surface wave [245], and wavefield modeling [95]. This very incomplete list over just one year period clearly demonstrates the wide-spread application of the DG method in computational science and engineering.

\section{Bound-preserving limiters}

In many convection dominated problems, the physical quantities have desired bounds which are satisfied by the exact solutions of the PDEs. For example, for two-dimensional incompressible Euler or Navier-Stokes equations written in a vorticity-streamfunction formulation, the vorticity satisfies a maximum principle. For Euler equations of compressible gas dynamics, density and pressure remain positive (non-negative) when their initial values are positive. It would certainly be desirable if numerical solutions obey the same bounds. If the numerical solution goes out of the bounds because of spurious oscillations, it would either be non-physical (e.g. negative density, negative internal energy, a percentage of a component which goes below zero or above one), or worse still, it could lead to nonlinear instability and blowups of the code because the PDE might become ill-posed (e.g. the Euler equations of compressible gas dynamics become ill-posed for negative density or pressure).

Most limiters in the literature are designed to control spurious oscillations. Not all of them can enforce the bound-preserving property. When they do, they might degenerate the order of accuracy of the original scheme in smooth regions.

Recently, a general framework is established by Zhang and Shu $[272,273]$ to preserve strict bounds (maximum principle for scalar problems and positivity of relevant quantities for

scalar problems or systems) for DG and finite volume schemes, while maintaining provable 
high order accuracy of the original schemes. These techniques apply to multi-dimensions in general unstructured triangulations as well [279]. For earlier work that this Zhang-Shu approach is based upon, we refer to [177].

We will not repeat here the details of this general framework and refer the readers to the references. We will only summarize here the main ingredients:

1. We start from a first order base DG or finite volume scheme (on first order level they are the same), using piecewise polynomials of degree zero (piecewise constants), which can be proved to be bound-preserving under certain CFL conditions for Euler forward time discretization.

For scalar hyperbolic conservation laws, the first order DG or finite volume scheme using any monotone numerical flux would satisfy a maximum principle. For Euler equations of compressible gas dynamics, several first order schemes, including the Godunov scheme [68], the Lax-Friedrichs scheme [177, 273], the Harten-Lax-van Leer (HLLE) scheme [94], and the Boltzmann type kinetic scheme [176], among others, are positivity-preserving for density and pressure. Modified HLLC first order solvers for multi-material compressible flow in the Lagrangian framework are shown to be positivity-preserving for density and internal energy for general equations of states in $[31,229,230]$. For relativistic hydrodynamics (RHD), the first order Lax-Friedrichs scheme is positivity-preserving for density and pressure, and maintains the upper bound of speed by that of the light $[244,182]$.

2. We then apply a simple scaling limiter to the high order DG polynomial, or the reconstructed polynomial for a finite volume scheme, at time level $n$. This scaling limiter is completely local, namely it uses information only within the current cell, not even its immediate neighbors. Also, its computation only involves the evaluation of the unlimited solution polynomial at a few pre-determined quadrature points, hence the cost is minimal. The limiter maintains the original cell average, thus ensuring conservation. 
3. We then evolve the solution by the Euler forward time discretization, or by TVD or SSP high order Runge-Kutta or multi-step time discretization [203, 207, 82, 81].

This procedure can be applied in arbitrary triangular meshes. Amazingly, this simple process guarantees bound-preserving under a fixed fraction of the CFL condition for the first order base scheme, as long as the bound-invariance region is convex in the phase space, and it leads to this mathematically provable bound-preserving property without degenerating the high order accuracy of the DG or finite volume schemes.

For scalar nonlinear conservation laws, passive convection in a divergence-free velocity field, and 2D incompressible Euler equations in the vorticity-streamfunction formulation, high order DG schemes maintaining maximum principle have been designed in [272, 279]. For Euler equations of gas dynamics, high order DG schemes maintaining positivity of density and pressure (or internal energy) have been designed in [273, 274, 275, 276, 279]. For shallow water equations, high order DG schemes maintaining non-negativity of water height have been designed in [248]. Positivity-preserving semi-Lagrangian DG schemes have been designed in $[183,195]$. Realizability-preserving DG and WENO schemes are constructed in $[2,198]$ for entropy based moment closures of linear kinetic equations. Positivity-preserving semi-implicit DG scheme is developed for solving the extended magnetohydrodynamics equations in [284]. Positivity-preserving Lagrangian schemes for multi-material compressible flows are designed in $[31,229,230]$. The positivity-preserving technique has allowed stable simulations of turbulent cosmology flows in [293]. Simulations for problems with $\delta$-function singularities, which are very difficult for high order methods, have been made possible by this positivity-preserving technique $[257,259]$. Bound-preserving high order DG methods have been designed for ideal MHD equations in [32] and for relativistic hydrodynamics (RHD) in [182]. The methodology can also be extended to schemes related to DG methods, such as the correction procedure via reconstruction $(\mathrm{CPR})$ method $[60,61]$.

While the Zhang-Shu approach mentioned above is simple and effective, it works most well for DG or finite volume schemes solving conservation laws. For finite difference schemes, 
this approach can also apply to, e.g. Euler equations to maintain positivity for density and pressure [277], but it has restrictions in order to keep the original high order accuracy. For convection-diffusion equations, this approach works for general DG methods to second order accuracy on arbitrary triangulations [282], and to third order accuracy for a special class of DG methods (the direct DG, or DDG methods) [30].

Another approach to achieve bound-preserving schemes is through the traditional fluxcorrection method, namely to put an explicit restriction on the high order numerical flux, often to make a convex combination of the high order flux and a first order bound-preserving flux, and choose the parameter carefully so that bound-preserving is ensured. The difficult task for this approach is to prove that the original high order accuracy is not compromised by this flux correction, and this is usually done via explicit and complicated algebraic verifications, thus limiting the scope that the proof can be applied. Among this class, we mention the high order parametrized maximum-principle-preserving and positivity-preserving finite difference schemes in $[253,148,249]$, and their extension to unstructured meshes in [37]. The limiters in [106] also belong to this class. Such parametrized positivity-preserving WENO schemes have been generalized to solve MHD equations in [38]. In [250], the limiter is extended to DG schemes for convection-diffusion equations, in [124], it is applied to high order finite difference WENO schemes solving correlated random walk with density-dependent turning rates, in [88], it is applied to DG schemes for nonlinear parabolic equations with blow-up solutions, and in [244], it is applied to finite difference WENO schemes for special relativistic hydrodynamics.

Although such parametrized maximum-principle-preserving and positivity-preserving limiters can also be applied to finite volume and DG schemes for conservation laws, their real advantage is for finite difference schemes solving conservation laws and schemes for solving convection-diffusion equations, for which the Zhang-Shu approach has rather severe restrictions as mentioned above. 


\section{WENO limiters for DG schemes}

Even though the DG method satisfies a cell-entropy inequality for the square entropy for both scalar nonlinear equations and symmetric nonlinear systems on arbitrary meshes $[119,102]$, which implies an $L^{2}$ or energy stability, this stability is not strong enough to prevent spurious oscillations or even blow-ups of the numerical solution in the presence of strong discontinuities. Therefore, nonlinear limiters are often needed to control such spurious oscillations.

Many limiters for the DG method exist in the literature, often adapted from those originally designed for finite volume schemes. Examples include the minmod type total variation bounded (TVB) limiter [202], applied to DG methods in [44, 43, 40, 45], the moment-based limiter [16] and the improved moment limiter [19]. Although these limiters can control spurious numerical oscillations near discontinuities, they tend to degrade accuracy when mistakenly used in smooth regions of the solution. It is usually difficult to design limiters to achieve both high order accuracy and a non-oscillatory property near discontinuities.

The limiters based on the weighted essentially non-oscillatory (WENO) methodology are designed with the objective of maintaining the original high order accuracy even if the limiters take effect in smooth cells. These limiters are based on the WENO methodology for finite volume schemes $[153,120]$, and involve nonlinear reconstructions of the polynomials in troubled cells using the information of neighboring cells. The WENO reconstructed polynomial has the same cell average and the same high order of accuracy as the original polynomial when the solution is smooth, and it is (essentially) non-oscillatory near discontinuities. In earlier years, Qiu and Shu [186] and Zhu et al. [289] designed WENO limiters using the usual WENO reconstruction based on cell averages of neighboring cells as in [120, 103, 201], to reconstruct the values of the solutions at certain Gaussian quadrature points in the target cells, and then rebuild the solution polynomials from the original cell average and the reconstructed values at the Gaussian quadrature points through a numerical integration for the moments. This limiter needs to use the information from not only the immediate neighboring cells but also neighbors' neighbors, making it complicated to implement in multi-dimensions, 
especially for unstructured meshes $[289,103,283]$. It also destroys the local data structure of the base DG scheme (which needs to communicate only with immediate neighbors), thus affecting its parallel efficiency. The effort in $[184,185]$ attempts to construct Hermite type WENO approximations, which use the information of not only the cell averages but also the lower order moments such as slopes, to reduce the spread of reconstruction stencils. However for higher order methods the information of neighbors' neighbors is still needed.

More recently, Zhong and Shu [285] developed a new WENO limiting procedure for Runge-Kutta DG methods on structured meshes. The idea is to reconstruct the entire polynomial, instead of reconstructing point values or moments in the classical WENO reconstructions. That is, the entire reconstruction polynomial on the target cell is a convex combination of polynomials on this cell and its immediate neighboring cells, with suitable adjustments for conservation and with the nonlinear weights of the convex combination following the classical WENO procedure. The main advantage of this limiter is its simplicity in implementation, as it uses only the information from immediate neighbors and the linear weights are always positive. This simplicity is more prominent for multi-dimensional unstructured meshes, which is studied in [290] for two-dimensional unstructured triangular meshes. Further improvements of this limiter are carried out in [291, 292]. This WENO limiter has also been adapted to the CPR schemes in $[60,61]$, which are closely related to DG schemes.

The WENO limiters are typically applied only in designated "troubled cells", in order to save computational cost and to minimize the influence to accuracy in smooth regions. Therefore, a troubled cell indicator is needed, to correctly identify cells near discontinuities in which the limiters should be applied. Qiu and Shu in [187] have compared several troubled cell indicators. In practice, the TVB indicator [202] and the KXRCF indicator [136] are often the best choices. We would also like to mention the recent troubled cell indicators based on wavelets and outlier detectors [232].

A novel sub-cell limiting which breaks the DG cell into subcells and then use WENO 
ideas for limiting is developed in [67], see also [265, 266].

\section{Inverse Lax-Wendroff type boundary conditions for finite difference schemes}

When a high order finite difference scheme with a wide stencil is used to solve partial differential equations, the inner schemes cannot be directly used near the boundary. Special treatments near the boundaries are needed in order to maintain accuracy and stability. There exist two difficulties when imposing numerical boundary conditions. Firstly, the points used in these schemes which lie outside the computational domain, namely the "ghost points", should be evaluated properly. Secondly, the grid points may not coincide with the physical boundary exactly, especially when Cartesian meshes are used to solve problems in complex geometry.

For hyperbolic conservation laws, classical Lagrangian extrapolation to evaluate ghost point values near the outflow boundary usually leads to stable approximations $[77,228,146]$. However, it is a challenge to obtain stable and accurate numerical boundary conditions near the inflow boundary. This is especially the case when the physical boundary does not coincide with but is very close to the first grid point, which is referred to as the "cut-cell" problem in the literature, see e.g. [14]. The inverse Lax-Wendroff (ILW) procedure, first introduced in [216], can overcome this difficulty. The simplified ILW (SILW) procedure, which is an extension of the ILW procedure and can save in algorithm complexity and computational cost, is introduced in [219]. For earlier related work, see [78, 79, 97, 113].

We would like to briefly discuss several different approaches in handling numerical boundary conditions for high order finite difference schemes, in order to put the ILW and SILW methods into proper perspective. One commonly used method to deal with complex geometry is to generate a boundary fitted mesh which allows us to impose boundary conditions directly in the algorithm. When employing this method, the governing equations are generally transformed into a new differential form on a curvilinear coordinate system (see e.g. 
[123]). If the domain is simple enough, a smooth mapping can be used for the transformation of the entire domain. But if the domain is more complex, composite overlapping meshes are usually generated to fit the physical boundaries, while these meshes are connected via interpolation (see e.g. [36, 99, 98, 199]). The disadvantage of this method is the complexity of generating the body-conformal grids. Another approach is to use Cartesian grids which do not conform to the physical boundary. The embedded boundary method is developed to solve the wave equation with Dirichlet or Neumann boundary conditions by using finite difference methods on Cartesian grids in $[134,135,133,170]$. In [210] the authors applied this method to hyperbolic conservation laws and obtained a second order accurate scheme. Baeza et al. [7] extended the approach from second order to fifth order using Lagrange extrapolation with a filter for the detection of discontinuities. A third approach is the so called immersed boundary method, which makes a modification of the original partial differential equations by introducing a forcing function at the physical boundary, and using it to reproduce the effects of boundary conditions, see, e.g. [178, 167].

The inverse Lax-Wendroff (ILW) method is similar to the immersed boundary method, but without introducing any forcing function to alter the original equations. In [216, 217, 218], Tan and Shu developed this high order accurate boundary treatment for hyperbolic conservation laws, based on the ILW procedure for the inflow boundaries and high order extrapolation for the outflow boundaries. This boundary treatment allows us to compute hyperbolic conservation laws defined on an arbitrary domain with a Cartesian mesh to arbitrary order of accuracy.

The main idea of the ILW procedure is repeatedly using the partial differential equation to convert the normal derivatives into the time derivatives and tangential derivatives at the physical boundary. With the giving inflow boundary condition and these normal derivatives, we can use Taylor expansions to assign values to grid points or ghost points near the physical boundary. A simplified ILW procedure which uses the ILW process only for the first few normal derivatives and then the less expensive high order extrapolation for the remaining 
ones is developed in [219], with good numerical results. Stability analysis for both the ILW and the simplified ILW procedures is given in [228, 146], proving that the method remains stable under the same CFL condition as that for problems without boundaries, regardless of the location of the first grid point relative to the location of the physical boundary. This indicates that the "cut cell" problem [14], which refers to the instability or the requirement of the extremely small CFL condition when the first grid point does not coincide with but is very close to the physical boundary, is effectively removed by the ILW or the simplified ILW procedure.

Let us use simple examples to show the ideas of the ILW procedure. Consider the hyperbolic equation

$$
\begin{aligned}
u_{t}+u_{x} & =0, \quad 0 \leq x<\infty \\
u(0, t) & =g(t)
\end{aligned}
$$

with a suitable initial condition. We take uniform mesh points as $0<x_{1}<x_{2}<\cdots$ where $x_{j+1}-x_{j}=\Delta x$ and $x_{1}=a \Delta x$ where $0<a<1$. Note that we have deliberately put the physical boundary $x=0$ not coinciding with the closest grid point $x_{1}$. Suppose we are using a fifth order upwind-biased scheme to approximate the derivative $u_{x}$, which involves a 6 -point stencil $x_{j-3}, x_{j-2}, \cdots, x_{j+2}$, then we would need to treat the first three points $x_{1}$, $x_{2}, x_{3}$ specially, or put values to the three ghost points $x_{0}, x_{-1}$ and $x_{-2}$ suitably, in order to apply the scheme. The ILW procedure to put values in these points or ghost points starts with a Taylor expansion

$$
u_{j}=\sum_{\ell=0}^{4} \frac{u^{(\ell)}(0)}{\ell !}\left(x_{j}\right)^{\ell}
$$

which is fifth order accurate, for $j=1,2,3$ (if we decide to put values to the first 3 grid points inside the domain) or for $j=0,-1,-2$ (if we decide to put values to the 3 ghost points outside the computational domain). The question is how to obtain the spatial derivatives $u^{(\ell)}(0)$ at the boundary $x=0$. We know that $u^{(0)}(0)=g(t)$ is given. Using the PDE (5.1), 
we can convert the first order spatial derivative $u^{(1)}(0)$ to first order time derivative

$$
u^{(1)}(0)=u_{x}(0, t)=-u_{t}(0, t)=-g^{\prime}(t)
$$

Likewise, by taking the time derivative of (5.1), we can convert the second order spatial derivative $u^{(2)}(0)$ to second order time derivative

$$
u^{(2)}(0)=u_{x x}(0, t)=u_{t t}(0, t)=g^{\prime \prime}(t)
$$

etc. Continuing in this process, we can convert all spatial derivatives at the physical boundary $x=0$ to time derivatives through repeatedly applying the PDE, hence we can evaluate the values of the grid points or ghost points (5.2) by using only the given boundary condition $u(0, t)=g(t)$. This is very similar to the traditional Lax-Wendroff scheme, in which the time derivatives are rewritten in terms of spatial derivatives through repeatedly using the PDE. Here the roles of time and space are reversed, hence the method was referred to as the inverse Lax-Wendroff procedure.

This procedure can be applied in the general case, up to multi-dimensional nonlinear systems, with the ILW procedure coupled with high order extrapolation for outflow boundary conditions. Stability can be proved $[228,146]$. Because the algebra may become very complicated for multi-dimensional nonlinear systems, a simplified ILW procedure, in which only the first few spatial derivatives are obtained by the ILW procedure and the remaining ones by high order extrapolation, is used in [219], with very good numerical results for the difficult problems of detonation computation. In [228, 146], stability analysis has been performed to exactly indicate how many spatial derivatives must be computed by the ILW procedure for different schemes in order to guarantee stability, regardless of the relative location of the first grid point to the physical boundary. This boundary treatment has been extended also to problems with moving boundaries [217] with very good performance.

So far, the ILW method has been mostly used on hyperbolic equations including conservation laws [216, 217, 218, 146] and Boltzmann type models [71], which involve only first order spatial derivatives in the equations. Very recently, Lu et al. extended this methodology 
to convection-diffusion equations [157]. It turns out that this extension is highly non-trivial, as totally different boundary treatments are needed for the diffusion-dominated and the convection-dominated regimes. A careful combination of these two boundary treatments has been designed in [157], in order to obtain a stable and accurate boundary condition for high order finite difference schemes when applied to convection-diffusion equations, regardless of whether they are convection or diffusion dominant.

The ILW and SILW methods have enabled simple finite difference schemes on Cartesian meshes to be used for solving complex geometry problems with shocked solutions [216, 217, 219, 157]. These methods hold a good potential in wider applications.

\section{Concluding remarks}

In this survey article we concentrate on two selected classes of high order methods, namely the weighted essentially non-oscillatory (WENO) finite difference and finite volume schemes and discontinuous Galerkin (DG) finite element methods, for solving time-dependent convectiondominated partial differential equations (PDEs). We first briefly review these methods with an indication of their general development and recent applications, and then describe three topics of their recent developments. The first is the development of bound-preserving limiters for DG, finite volume and finite difference schemes, emphasizing the ability of high order schemes to maintain physical bounds without compromising their high order accuracy. The second is the WENO limiters for DG methods, which address issues in non-oscillatory performance when there are strong shocks. Finally, the third topic is an inverse Lax-Wendroff type boundary treatments for finite difference schemes on Cartesian meshes to solve PDEs in complex geometry. While details have been omitted to save space, references are given for readers interested in these developments to find them. We hope this survey paper gives evidence that high order accuracy methods are among the good choices for solving convection-dominated PDEs in science and engineering, and would thus encourages more researchers to study and apply these methods. 


\section{References}

[1] R. Abgrall, On essentially non-oscillatory schemes on unstructured meshes: analysis and implementation, Journal of Computational Physics, 114 (1994), 45-58.

[2] G. Alldredge and F. Schneider, A realizability-preserving discontinuous Galerkin scheme for entropy-based moment closures for linear kinetic equations in one space dimension, Journal of Computational Physics, 295 (2015), 665-684.

[3] L. Almazan, D. Serero, C. Saluena and T. Poschel, Self-organized shocks in the sedimentation of a granular gas, Physical Review E, 91 (2015), 062214.

[4] M. Atak, A. Beck, T. Bolemann, D. Flad, H. Frank, F. Hindenlang and C.-D. Munz, Discontinuous Galerkin for high performance computational fluid dynamics, in W. Nagel, D. Kroner and M. Resch, Eds, High Performance Computing in Science and Engineering 14, Springer, 2015, 499-518.

[5] M. Baccouch, Asymptotically exact a posteriori local discontinuous Galerkin error estimates for the one-dimensional second-order wave equation, Numerical Methods for Partial Differential Equations, 31 (2015), 1461-1491.

[6] M. Baccouch and S. Adjerid, A posteriori local discontinuous Galerkin error estimation for two-dimensional convection-diffusion problems, Journal of Scientific Computing, 62 (2015), 399-430.

[7] A. Baeza, P. Mulet and D. Zoro, High order accurate extrapolation technique for finite difference methods on complex domains with Cartesian meshes, Journal of Scientific Computing, 66 (2016), 761-791.

[8] D.S. Balsara and C.-W. Shu, Monotonicity preserving weighted essentially nonoscillatory schemes with increasingly high order of accuracy, Journal of Computational Physics, 160 (2000), 405-452. 
[9] F. Bassi and S. Rebay, A high-order accurate discontinuous finite element method for the numerical solution of the compressible Navier-Stokes equations, Journal of Computational Physics, 131 (1997), 267-279.

[10] C.E. Baumann and J.T. Oden, A discontinuous hp finite element method for convection-diffusion problems, Computer Methods in Applied Mechanics and Engineering, 175 (1999), 311-341.

[11] G. Bechon, R. Mevel, D. Davidenko and J.E. Shepherd, Modeling of Rayleigh scattering imaging of detonation waves: Quantum computation of Rayleigh cross-sections and real diagnostic effects, Combustion and Flame, 162 (2015), 2191-2199.

[12] A.D. Beck, T. Bolemann, D. Flad, H. Frank, G.J. Gassner, F. Hindenlang and C.D. Munz, High-order discontinuous Galerkin spectral element methods for transitional and turbulent flow simulations, International Journal for Numerical Methods in Fluids, 76 (2014), 522-548.

[13] S. Beig and E. Johnsen, Maintaining interface equilibrium conditions in compressible multiphase flows using interface capturing, Journal of Computational Physics, 302 (2015), 548-566.

[14] M.J. Berger, C. Helzel and R.J. Leveque, h-box methods for the approximation of hyperbolic conservation laws on irregular grids, SIAM Journal on Numerical Analysis, 41 (2003), 893-918.

[15] F. Bisetti, S.M. Sarathy, M. Toma and S.H. Chung, Stabilization and structure of n-heptane tribrachial flames in axisymmetric laminar jets, Proceedings of the Combustion Institute, 35 (2015), 1023-1032.

[16] R. Biswas, K.D. Devine and J. Flaherty, Parallel, adaptive finite element methods for conservation laws, Applied Numerical Mathematics, 14 (1994), 255-283. 
[17] O. Bokanowski, Y. Cheng and C.-W. Shu, Convergence of discontinuous Galerkin schemes for front propagation with obstacles, Mathematics of Computation, to appear. DOI: http://dx.doi.org/10.1090/mcom/3072

[18] R. Borges, M. Carmona, B. Costa, W.S. Don, An improved weighted essentially nonoscillatory scheme for hyperbolic conservation laws, Journal of Computational Physics, 227 (2008), 3191-3211.

[19] A. Burbeau, P. Sagaut and Ch.H. Bruneau, A problem-independent limiter for high-order Runge-Kutta discontinuous Galerkin methods, Journal of Computational Physics, 169 (2001), 111-150.

[20] B. Burkhart, A. Lazarian, D. Balsara, C. Meyer and J. Cho, Alfvenic turbulence beyond the ambipolar diffusion scale, Astrophysical Journal, 805 (2015), 118.

[21] S. Canic, B. Piccoli, J.-M. Qiu and T. Ren, Runge-Kutta discontinuous Galerkin method for traffic flow model on networks, Journal of Scientific Computing, 63 (2015), $233-255$.

[22] W. Cao, C.-W. Shu, Y. Yang and Z. Zhang, Superconvergence of discontinuous Galerkin methods for 2-D hyperbolic equations, SIAM Journal on Numerical Analysis, 53 (2015), 1651-1671.

[23] W. Cao, Z. Zhang and Q. Zou, Superconvergence of discontinuous Galerkin method for linear hyperbolic equations, SIAM Journal on Numerical Analysis, 52 (2014), 25552573.

[24] H. Carlsson, R. Yu and X.-S. Bai, Flame structure analysis for categorization of lean premixed $\mathrm{CH}_{4} /$ air and $\mathrm{H}$-2/air flames at high Karlovitz numbers: Direct numerical simulation studies, Proceedings of the Combustion Institute, 35 (2015), 1425-1432. 
[25] J. Casper, C.-W. Shu and H. Atkins, Comparison of two formulations for high-order accurate essentially nonoscillatory schemes, AIAA Journal, 32 (1994), 1970-1977.

[26] M. Castro, B. Costa and W.S. Don, High order weighted essentially non-oscillatory WENO-Z schemes for hyperbolic conservation laws, Journal of Computational Physics, 230 (2011), 1766-1792.

[27] O. Cessenat and B. Després, Application of an ultra weak variational formulation of elliptic PDEs to the two-dimensional Helmholtz equation, SIAM Journal on Numerical Analysis, 35 (1998), 255-299.

[28] M.A. Chella, H. Bihs and D. Myrhaug, Characteristics and profile asymmetry properties of waves breaking over an impermeable submerged reef, Coastal Engineering, 100 (2015), 26-36.

[29] M.A. Chella, H. Bihs, D. Myrhaug and M. Muskulus, Breaking characteristics and geometric properties of spilling breakers over slopes, Coastal Engineering, 95 (2015), 4-19.

[30] Z. Chen and J. Yan, Third order maximum-principle-satisfying direct discontinuous Galerkin methods for time dependent convection diffusion equations on unstructured triangular meshes, Journal of Computational Physics, 308 (2016), 198-217.

[31] J. Cheng and C.-W. Shu, Positivity-preserving Lagrangian scheme for multi-material compressible flow, Journal of Computational Physics, 257 (2014), 143-168.

[32] Y. Cheng, F. Li, J. Qiu and L. Xu, Positivity-preserving DG and central DG methods for ideal MHD equations, Journal of Computational Physics, 238 (2013), 255-280.

[33] Y. Cheng and C.-W. Shu, A discontinuous Galerkin finite element method for time dependent partial differential equations with higher order derivatives, Mathematics of Computation, 77 (2008), 699-730. 
[34] Y. Cheng and C.-W. Shu, Superconvergence and time evolution of discontinuous Galerkin finite element solutions, Journal of Computational Physics, 227 (2008), 96129627.

[35] Y. Cheng and C.-W. Shu, Superconvergence of discontinuous Galerkin and local discontinuous Galerkin schemes for linear hyperbolic and convection-diffusion equations in one space dimension, SIAM Journal on Numerical Analysis, 47 (2010), 4044-4072.

[36] G. Chesshire and W.D. Henshaw, Composite overlapping meshes for the solution of partial differential equations, Journal of Computational Physics, 90 (1990), 1-64.

[37] A.J. Christlieb, Y. Liu, Q. Tang and Z. Xu, High order parametrized maximumprinciple-preserving and positivity-preserving WENO schemes on unstructured meshes, Journal of Computational Physics, 281 (2015), 334-351.

[38] A.J. Christlieb, Y. Liu, Q. Tang and Z. Xu, Positivity-preserving finite difference weighted ENO schemes with constrained transport for ideal magnetohydrodynamic equations, SIAM Journal on Scientific Computing, 37 (2015), A1825-A1845.

[39] B. Cockburn, Discontinuous Galerkin methods for convection-dominated problems, in High-Order Methods for Computational Physics, T.J. Barth and H. Deconinck (eds.), Lecture Notes in Computational Science and Engineering, volume 9, Springer, 1999, pp.69-224.

[40] B. Cockburn, S. Hou and C.-W. Shu, The Runge-Kutta local projection discontinuous Galerkin finite element method for conservation laws IV: the multidimensional case, Mathematics of Computation, 54 (1990), 545-581.

[41] B. Cockburn, G. Karniadakis and C.-W. Shu, The development of discontinuous Galerkin methods, in Discontinuous Galerkin Methods: Theory, Computation and Applications, B. Cockburn, G. Karniadakis and C.-W. Shu (eds.), Lecture Notes in 
Computational Science and Engineering, volume 11, Springer, 2000, Part I: Overview, pp.3-50.

[42] B. Cockburn, F. Li and C.-W. Shu, Locally divergence-free discontinuous Galerkin methods for the Maxwell equations, Journal of Computational Physics, 194 (2004), 588-610.

[43] B. Cockburn, S.-Y. Lin and C.-W. Shu, TVB Runge-Kutta local projection discontinuous Galerkin finite element method for conservation laws III: one-dimensional systems, Journal of Computational Physics, 84 (1989), 90-113.

[44] B. Cockburn and C.-W. Shu, TVB Runge-Kutta local projection discontinuous Galerkin finite element method for conservation laws II: general framework, Mathematics of Computation, 52 (1989), 411-435.

[45] B. Cockburn and C.-W. Shu, The Runge-Kutta discontinuous Galerkin method for conservation laws $V$ : multidimensional systems, Journal of Computational Physics, 141 (1998), 199-224.

[46] B. Cockburn and C.-W. Shu, The local discontinuous Galerkin method for timedependent convection-diffusion systems, SIAM Journal on Numerical Analysis, 35 (1998), 2440-2463.

[47] B. Cockburn and C.-W. Shu, Runge-Kutta discontinuous Galerkin methods for convection-dominated problems, Journal of Scientific Computing, 16 (2001), 173-261.

[48] B. Cockburn and C.-W. Shu, Foreword for the special issue on discontinuous Galerkin method, Journal of Scientific Computing, 22-23 (2005), 1-3.

[49] B. Cockburn and C.-W. Shu, Foreword for the special issue on discontinuous Galerkin method, Journal of Scientific Computing, 40 (2009), 1-3. 
[50] C. Dawson, Foreword for the special issue on discontinuous Galerkin method, Computer Methods in Applied Mechanics and Engineering, 195 (2006), 3183.

[51] M.T.H. de Frahan and E. Johnsen, Numerical simulations of a shock interacting with successive interfaces using the discontinuous Galerkin method: the multilayered Richtmyer-Meshkov and Rayleigh-Taylor instabilities, Shock Waves, 25 (2015), 329345.

[52] M.T.H. de Frahan, S. Varadan and E. Johnsen, A new limiting procedure for discontinuous Galerkin methods applied to compressible multiphase flows with shocks and interfaces, Journal of Computational Physics, 280 (2015), 489-509.

[53] S. Delcourte and N. Glinsky, Analysis of a high-order space and time discontinuous Galerkin method for elastodynamic equations. Application to 3D wave propagation, ESAIM: Mathematical Modelling and Numerical Analysis $\left(M^{2} A N\right), 49$ (2015), 10851126.

[54] X. Deng and H. Zhang, Developing high-order weighted compact nonlinear schemes, Journal of Computational Physics, 165 (2000), 22-44.

[55] T. Dietrich, S. Bernuzzi, M. Ujevic and B. Brugmann, Numerical relativity simulations of neutron star merger remnants using conservative mesh refinement, Physical Review D, 91 (2015), 124041.

[56] D.A Di Pietro and A. Ern, Mathematical Aspects of Discontinuous Galerkin Methods, Springer, Berlin Heidelberg, 2012.

[57] W.S. Don and R. Borges, Accuracy of the weighted essentially non-oscillatory conservative finite difference schemes, Journal of Computational Physics, 250 (2013), 347-372. 
[58] B. Dong, C.-W. Shu and W. Wang, A new multiscale discontinuous Galerkin method for the one-dimensional stationary Schrödinger equation, Journal of Scientific Computing, 66 (2016), 321-345.

[59] A. Dorda and F. Schuerrer, A WENO-solver combined with adaptive momentum discretization for the Wigner transport equation and its application to resonant tunneling diodes, Journal of Computational Physics, 284 (2015), 95-116.

[60] J. Du, C.-W. Shu and M. Zhang, A simple weighted essentially non-oscillatory limiter for the correction procedure via reconstruction (CPR) framework, Applied Numerical Mathematics, 95 (2015), 173-198.

[61] J. Du, C.-W. Shu and M. Zhang, A simple weighted essentially non-oscillatory limiter for the correction procedure via reconstruction (CPR) framework on unstructured meshes, Applied Numerical Mathematics, 90 (2015), 146-167.

[62] M. Dumbser and D.S. Balsara, High-order unstructured one-step PnPm schemes for the viscous and resistive MHD equations, CMES - Computer modeling in Engineering \& Sciences, 54 (2009), 301-333.

[63] M. Dumbser, D.S. Balsara, E.F. Toro and C.D. Munz, A unified framework for the construction of one-step finite volume and discontinuous Galerkin schemes on unstructured meshes, Journal of Computational Physics, 227 (2008), 8209-8253.

[64] M. Dumbser and M. Facchini, A space-time discontinuous Galerkin method for Boussinesq-type equations, Applied Mathematics and Computation, 272 (2016), 336346.

[65] M. Dumbser, A. Hidalgo and O. Zanotti, High order space time adaptive ADER-WENO finite volume schemes for non-conservative hyperbolic systems, Computer Methods in Applied Mechanics and Engineering, 268 (2014), 359-387. 
[66] M. Dumbser and M. Käser, Arbitrary high order non-oscillatory finite volume schemes on unstructured meshes for linear hyperbolic systems, Journal of Computational Physics, 221 (2007), 693-723.

[67] M. Dumbser, O. Zanotti, R. Loubere and S. Diot, A posteriori subcell limiting of the discontinuous Galerkin finite element method for hyperbolic conservation laws, Journal of Computational Physics, 278 (2014), 47-75.

[68] B. Einfeldt, C.D. Munz, P.L. Roe and B. Sjögreen, On Godunov-Type methods near low densities, Journal of Computational Physics, 92 (1991), 273-295.

[69] D. Elfverson, E.H. Georgoulis and A. Malqvist, An adaptive discontinuous Galerkin multiscale method for elliptic problems, Multiscale Modeling and Simulation, 11 (2013), 747-765.

[70] A.O. Fierro, E.R. Mansell, C.L. Ziegler and D.R. MacGorman, Explicitly simulated electrification and lightning within a tropical cyclone based on the environment of hurricane Isaac (2012), Journal of the Atmospheric Sciences, 72 (2015), 4167-4193.

[71] F. Filbet and C. Yang, An inverse Lax-Wendroff method for boundary conditions applied to Boltzmann type models, Journal of Computational Physics, 245 (2013), 43-61.

[72] O. Friedrichs, Weighted essentially non-oscillatory schemes for the interpolation of mean values on unstructured grids, Journal of Computational Physics, 144 (1998), $194-212$.

[73] N. Gerhard, D. Caviedes-Voullieme, S. Muller and G. Kesserwani, Multiwavelet-based grid adaptation with discontinuous Galerkin schemes for shallow water equations, Journal of Computational Physics, 301 (2015), 265-288.

[74] G.A. Gerolymos and I. Vallet, Reynolds-stress model prediction of 3-D duct flows, Flow Turbulence and Combustion, 96 (2016), 45-93. 
[75] N.S. Ghaisas, D.A. Shetty and S.H. Frankel, Large eddy simulation of turbulent horizontal buoyant jets, Journal of Turbulence, 16 (2015), 772-808.

[76] C.J. Gittelson, R. Hiptmair and I. Perugia, Plane wave discontinuous Galerkin methods: analysis of the h-version, ESAIM: Mathematical Modelling and Numerical Analysis $\left(M^{2} A N\right), 43$ (2009), 297-331.

[77] M. Goldberg, On a boundary extrapolation theorem by Kreiss. Mathematics of Computation, 31 (1977), 469-477.

[78] M. Goldberg and E. Tadmor, Scheme-independent stability criteria for difference approximations of hyperbolic initial-boundary value problems. I, Mathematics of Computation, 32 (1978), 1097-1107.

[79] M. Goldberg and E. Tadmor, Scheme-independent stability criteria for difference approximations of hyperbolic initial-boundary value problems. II. Mathematics of Computation, 36 (1981), 603-626.

[80] P. Gonzalez, M.J. Ibanez, A.M. Roldan and J.B. Roldan, An in-depth study on WENObased techniques to improve parameter extraction procedures in MOSFET transistors, Mathematics and Computers in Simulation, 118 (2015), 248-257.

[81] S. Gottlieb, D. Ketcheson and C.-W. Shu, Strong Stability Preserving Runge-Kutta and Multistep Time Discretizations, World Scientific, Singapore, 2011.

[82] S. Gottlieb, C.-W. Shu and E. Tadmor, Strong stability-preserving high-order time discretization methods, SIAM Review, 43 (2001), 89-112.

[83] H. Grimm-Strele, F. Kupka, B. Low-Baselli, E. Mundprecht, F. Zaussinger and P. Schiansky, Realistic simulations of stellar surface convection with ANTARES: I. Boundary conditions and model relaxation, New Astronomy, 34 (2015), 278-293. 
[84] H. Guo, Q. Zhang and J. Wang, Error analysis of the semi-discrete local discontinuous Galerkin method for compressible miscible displacement problem in porous media, Applied Mathematics and Computation, 259 (2015), 88-105.

[85] L. Guo and Y. Xu, An efficient, unconditionally energy stable local discontinuous Galerkin scheme for the Cahn-Hilliard-Brinkman system, Journal of Computational Physics, 298 (2015), 387-405.

[86] L. Guo and Y. Xu, Energy conserving local discontinuous Galerkin methods for the nonlinear Schrodinger equation with wave operator, Journal of Scientific Computing, $65(2015), 622-647$.

[87] L. Guo, Y. Xu and Z. Xu, Local discontinuous Galerkin methods for the functionalized Cahn-Hilliard equation, Journal of Scientific Computing, 63 (2015), 913-937

[88] L. Guo and Y. Yang, Positivity preserving high-order local discontinuous Galerkin method for parabolic equations with blow-up solutions, Journal of Computational Physics, 289 (2015), 181-195.

[89] T. Haga, H. Gao and Z.J. Wang, A high-order unifying discontinuous formulation for the Navier-Stokes equations on 3D mixed grids. Mathematical Modelling of Natural Phenomena, 6 (2011), 28-56.

[90] M. Hajipour and A. Malek, High accurate modified WENO method for the solution of Black-Scholes equation, Computational and Applied Mathematics, 34 (2015), 125-140.

[91] L.H. Han, X.Y. Hu and N.A. Adams, Scale separation for multi-scale modeling of freesurface and two-phase flows with the conservative sharp interface method, Journal of Computational Physics, 280 (2015), 387-403. 
[92] W. Han, Y. Gao, C. Wang and C.K. Law, Coupled pulsating and cellular structure in the propagation of globally planar detonations in free space, Physics of Fluids, 27 (2015), 106101.

[93] A. Harten, B. Engquist, S. Osher and S. Chakravarthy, Uniformly high order essentially non-oscillatory schemes, III, Journal of Computational Physics, 71 (1987), 231-303.

[94] A. Harten, P.D. Lax and B. van Leer, On upstream differencing and Godunov type schemes for hyperbolic conservation laws, SIAM Review, 25 (1983), 35-61.

[95] X. He, D. Yang and H. Wu, A weighted Runge-Kutta discontinuous Galerkin method for wavefield modelling, Geophysical Journal International, 200 (2015), 1389-1410.

[96] A.K. Henrick, T.D. Aslam and J.M. Powers, Mapped weighted essentially nonoscillatory schemes: Achieving optimal order near critical points, Journal of Computational Physics, 207 (2005), 542-567.

[97] W.D. Henshaw, A high-order accurate parallel solver for Maxwell's equations on overlapping grids, SIAM Journal on Scientific Computing, 28 (2006), 1730-1765.

[98] W.D. Henshaw and K.K. Chand, A composite grid solver for conjugate heat transfer in fluid-structure systems, Journal of Computational Physics, 228 (2009), 3708-3741.

[99] W.D. Henshaw, H.-O. Kreiss and L.G.M. Reyna, A fourth-order accurate difference approximation for the incompressible Navier-Stokes equations, Computers \& Fluids, 23 (1994), 575-593.

[100] J. Hesthaven and T. Warburton, Nodal Discontinuous Galerkin Methods, Springer, New York, 2008.

[101] A. Holmqvist, C. Andersson, F. Magnusson and J. Akesson, Methods and tools for robust optimal control of batch chromatographic separation processes, Processes, 3 (2015), 568-606. 
[102] S. Hou and X.-D. Liu, Solutions of multi-dimensional hyperbolic systems of conservation laws by square entropy condition satisfying discontinuous Galerkin method, Journal of Scientific Computing, 31 (2007), 127-151.

[103] C. Hu and C.-W. Shu, Weighted essentially non-oscillatory schemes on triangular meshes, Journal of Computational Physics, 150 (1999), 97-127.

[104] F. Hu, R. Wang, X. Chen and H. Feng, An adaptive mesh method for 1D hyperbolic conservation laws, Applied Numerical Mathematics, 91 (2015), 11-25.

[105] H. Hu and C. Chen, Superconvergence of bi-k degree time-space fully discontinuous finite element for first-order hyperbolic equations, Advances in Applied Mathematics and Mechanics, 7 (2015), 323-337.

[106] X.Y. Hu, N.A. Adams and C.-W. Shu, Positivity-preserving method for high-order conservative schemes solving compressible Euler equations, Journal of Computational Physics, 242 (2013), 169-180.

[107] X.Y. Hu, B. Wang and N.A. Adams, An efficient low-dissipation hybrid weighted essentially non-oscillatory scheme, Journal of Computational Physics, 301 (2015), 415-424.

[108] Y. Hu, Q. Shi, V.F. De Almeida and X. Li, Numerical simulation of phase transition problems with explicit interface tracking, Chemical Engineering Science, 128 (2015), 92-108.

[109] Z.M. Hu, C. Wang, Z.L. Jiang and B.C. Khoo, On the numerical technique for the simulation of hypervelocity test flows, Computers \& Fluids, 106 (2015), 12-18.

[110] G. Huber, S. Tanguy, J.-C. Bera and B. Gilles, A time splitting projection scheme for compressible two-phase flows. Application to the interaction of bubbles with ultrasound waves, Journal of Computational Physics, 302 (2015), 439-468. 
[111] C. Huang, X. Yu, C. Wang, Z. Li and N. An, A numerical method based on fully discrete direct discontinuous Galerkin method for the time fractional diffusion equation, Applied Mathematics and Computation, 264 (2015), 483-492.

[112] C.-H. Huang, C.-C. Lin and M.-S. Ju, Discontinuous Galerkin finite element method for solving population density functions of cortical pyramidal and thalamic neuronal populations, Computers in Biology and Medicine, 57 (2015), 150-158.

[113] L. Huang, C.-W. Shu and M. Zhang, Numerical boundary conditions for the fast sweeping high order WENO methods for solving the Eikonal equation, Journal of Computational Mathematics, 26 (2008), 336-346.

[114] T. Huang, W. Tian, Y. Zhang, G. Su, S. Qiu, X. Yang and H. Yin, Development of DETAC and its application to the hydrogen detonation analysis, Progress in Nuclear Energy, 85 (2015), 228-238.

[115] T. Huttunen, P. Monk and J. Kaipio, Computational aspects of the ultra-weak variational formulation, Journal of Computational Physics, 182 (2002), 27-46.

[116] H.T. Huynh, A flux reconstruction approach to high-order schemes including discontinuous Galerkin methods, AIAA Paper 2007-4079.

[117] J.Y. Hwang and O.J. Kwon, Assessment of tip shape effect on rotor aerodynamic performance in hover, International Journal of Aeronautical and Space Sciences, 16 (2015), 295-310.

[118] F. Jia, Z. Gao and W.S. Don, A spectral study on the dissipation and dispersion of the WENO schemes, Journal of Scientific Computing, 63 (2015), 49-77.

[119] G.-S. Jiang and C.-W. Shu, On cell entropy inequality for discontinuous Galerkin methods, Mathematics of Computation, 62 (1994), 531-538. 
[120] G.-S. Jiang and C.-W. Shu, Efficient implementation of weighted ENO schemes, Journal of Computational Physics, 126 (1996), 202-228.

[121] L. Jiang, H. Shan and C.Q. Liu, Weighted compact scheme, International Journal of Computational Fluid Dynamics, 15 (2001), 147-155.

[122] Y. Jiang, C.-W. Shu and M. Zhang, An alternative formulation of finite difference weighted ENO schemes with Lax-Wendroff time discretization for conservation laws, SIAM Journal on Scientific Computing, 35 (2013), A1137-A1160.

[123] Y. Jiang, C.-W. Shu and M. Zhang, Free-stream preserving finite difference schemes on curvilinear meshes, Methods and Applications of Analysis, 21 (2014), 1-30.

[124] Y. Jiang, C.-W. Shu and M. Zhang, High order finite difference WENO schemes with positivity-preserving limiter for correlated random walk with density-dependent turning rates, Mathematical Models and Methods in Applied Sciences $\left(M^{3} A S\right), 25$ (2015), 1553-1588.

[125] A. Kamath, M.A. Chella, H. Bihs and O.A. Arntsen, CFD investigations of wave interaction with a pair of large tandem cylinders, Ocean Engineering, 108 (2015), 738748.

[126] G. Kanschat, Discontinuous Galerkin Methods for Viscous Flow, Deutscher Universitätsverlag, Wiesbaden, 2007.

[127] O. Karakashian and C. Makridakis, A posteriori error estimates for discontinuous Galerkin methods for the generalized Korteweg-de Varies equation, Mathematics of Computation, 84 (2015), 1145-1167.

[128] K.K. Katta, R.D. Kiran and V. Kumar, High-order finite volume shallow water model on the cubed-sphere: 1D reconstruction scheme, Applied Mathematics and Computation, 266 (2015), 316-327. 
[129] A. Khosronejad, J.L. Kozarek, M.L. Palmsten and F. Sotiropoulos, Numerical simulation of large dunes in meandering streams and rivers with in-stream rock structures, Advances in Water Resources, 81 (2015), 45-61.

[130] A. Klockner, T. Warburton, J. Bridge and J. Hesthaven, Nodal discontinuous Galerkin methods on graphics processors, Journal of Computational Physics, 228 (2010), 78637882.

[131] I.W. Kokkinakis and D. Drikakis, Implicit large eddy simulation of weakly-compressible turbulent channel flow, Computer Methods in Applied Mechanics and Engineering, 287 (2015), 229-261.

[132] D.A. Kopriva and J.H. Kolias, A conservative staggered-grid Chebyshev multidomain method for compressible flows, Journal of Computational Physics, 125 (1996), 244-261.

[133] H.-O. Kreiss and N.A. Petersson, A second order accurate embedded boundary method for the wave equation with Dirichlet data, SIAM Journal on Scientific Computing, 27 (2006), 1141-1167.

[134] H.-O. Kreiss, N.A. Petersson and J. Yström, Difference approximations for the second order wave equation, SIAM Journal on Numerical Analysis, 40 (2002), 1940-1967.

[135] H.-O. Kreiss, N.A. Petersson and J. Yström, Difference approximations of the Neumann problem for the second order wave equation, SIAM Journal on Numerical Analysis, 42 (2004), 1292-1323.

[136] L. Krivodonova, J. Xin, J.-F. Remacle, N. Chevaugeon and J.E. Flaherty, Shock detection and limiting with discontinuous Galerkin methods for hyperbolic conservation laws, Applied Numerical Mathematics, 48 (2004), 323-338.

[137] F. Kupka, M. Losch, F. Zaussinger and T. Zweigle, Semi-convection in the ocean and in stars: A multi-scale analysis, Meteorologische Zeitschrift, 24 (2015), 343-358. 
[138] D. Lannesa and F. Marche, A new class of fully nonlinear and weakly dispersive GreenNaghdi models for efficient 2D simulations, Journal of Computational Physics, 282 (2015), 238-268.

[139] S.K. Lele, Compact finite difference schemes with spectral-like resolution, Journal of Computational Physics, 103 (1992), 16-42.

[140] S.-C. Leung, M.-C. Chu and L.-M. Lin, A new hydrodynamics code for Type Ia supernovae, Monthly Notices of the Royal Astronomical Society, 454 (2015), 1238-1259.

[141] B. Li, Discontinuous Finite Elements in Fluid Dynamics and Heat Transfer, Birkhauser, Basel, 2006.

[142] F. Li and C.-W. Shu, Locally divergence-free discontinuous Galerkin methods for MHD equations, Journal of Scientific Computing, 22-23 (2005), 413-442.

[143] F. Li and C.-W. Shu, Reinterpretation and simplified implementation of a discontinuous Galerkin method for Hamilton-Jacobi equations, Applied Mathematics Letters, 18 (2005), 1204-1209.

[144] H. Li, S. Do and M. Kang, A wavelet-based adaptive WENO algorithm for Euler equations, Computers \& Fluids, 123 (2015), 10-22.

[145] J. Li, J. Ning and J.H.S. Lee, Mach reflection of a ZND detonation wave, Shock Waves, 25 (2015), 293-304.

[146] T. Li, C.-W. Shu and M. Zhang, Stability analysis of the inverse Lax-Wendroff boundary treatment for high order upwind-biased finite difference schemes, Journal of Computational and Applied Mathematics, 299 (2016), 140-158.

[147] Z. Li, A.-P. Peng, H.-X. Zhang and J.-Y. Yang, Rarefied gas flow simulations using high-order gas-kinetic unified algorithms for Boltzmann model equations, Progress in Aerospace Sciences, 74 (2015), 81-113. 
[148] C. Liang and Z. Xu, Parametrized maximum-principle-preserving flux limiters for high order schemes solving multi-dimensional scalar hyperbolic conservation laws, Journal of Scientific Computing, 58 (2014), 41-60.

[149] X. Liang, A.Q.M. Khaliq and Y. Xing, Fourth order exponential time differencing method with local discontinuous Galerkin approximation for coupled nonlinear Schrodinger equations, Communications in Computational Physics, 17 (2015), 510-541.

[150] X. Liang and X. Li, Direct numerical simulation on Mach number and wall temperature effects in the turbulent flows of flat-plate boundary layer, Communications in Computational Physics, 17 (2015), 189-212.

[151] H. Liu and J. Qiu, Finite difference Hermite WENO schemes for conservation laws, II: an alternative approach, Journal of Scientific Computing, 66 (2016), 598-624.

[152] H. Liu and H. Yu, The entropy satisfying discontinuous Galerkin method for FokkerPlanck equations, Journal of Scientific Computing, 62 (2015), 803-830.

[153] X. Liu, S. Osher and T. Chan, Weighted essentially non-oscillatory schemes, Journal of Computational Physics, 115 (1994), 200-212.

[154] X. Liu, S. Zhang, H. Zhang and C.-W. Shu, A new class of central compact schemes with spectral-like resolution II: Hybrid weighted nonlinear schemes, Journal of Computational Physics, 284 (2015), 133-154.

[155] Y. Liu and C.-W. Shu, Analysis of the local discontinuous Galerkin method for the drift-diffusion model of semiconductor devices, Science China Mathematics, 59 (2016), $115-140$.

[156] Y. Liu, M. Vinokur and Z.J. Wang, Spectral difference method for unstructured grids I: Basic formulation, Journal of Computational Physics, 216 (2006), 780-801. 
[157] J. Lu, J. Fang, S. Tan, C.-W. Shu and M. Zhang, Inverse Lax-Wendroff procedure for numerical boundary conditions of convection-diffusion equations, submitted to Journal of Computational Physics.

[158] W. Lu, Y. Huang and H. Liu, Mass preserving discontinuous Galerkin methods for Schrodinger equations, Journal of Computational Physics, 282 (2015), 210-226.

[159] F. Luddens, M. Bergmann and L. Weynans, Enablers for high-order level set methods in fluid mechanics, International Journal for Numerical Methods in Fluids, 79 (2015), 654-675.

[160] J. Luo, X.Y. Hu and A. Adams, A conservative sharp interface method for incompressible multiphase flows, Journal of Computational Physics, 284 (2015), 547-565.

[161] M.P. Martin, E.M. Taylor, M. Wu and V.G. Weirs, A bandwidth-optimized WENO scheme for the effective direct numerical simulation of compressible turbulence, Journal of Computational Physics, 220 (2006), 270-289.

[162] C. Meng, L. Wang, Z. Cao, L.-L. Feng and W. Zhu, Large-scale parallelization based on CPU and GPU cluster for cosmological fluid simulations, Computers \& Fluids, 110 (2015), 152-158.

[163] X. Meng, C.-W. Shu, Q. Zhang and B. Wu, Superconvergence of discontinuous Galerkin method for scalar nonlinear conservation laws in one space dimension, SIAM Journal on Numerical Analysis, 50 (2012), 2336-2356.

[164] R. Mevel, D. Davidenko, F. Lafosse, N. Chaumeix, G. Dupre, C.-E. Paillard, and J.E. Shepherd, Detonation in hydrogen-nitrous oxide-diluent mixtures: An experimental and numerical study, Combustion and Flame, 162 (2015), 1638-1649. 
[165] D. Min, W. Wang and S. Li, Numerical analysis of space charge accumulation and conduction properties in LDPE nanodielectrics, IEEE Transactions on Dielectrics and Electrical Insulation, 22 (2015), 1483-1491.

[166] T. Minoshima, S. Hirose and T Sano, Dependence of the saturation level of magnetorotational instability on gas pressure and magnetic Prandtl number, Astrophysical Journal, 808 (2015), 54 .

[167] R. Mittal and G. Iaccarino, Immersed boundary methods, Annual Review of Fluid Mechanics, 37 (2005), 239-261.

[168] L.O. Muller and P.J. Blanco, A high order approximation of hyperbolic conservation laws in networks: Application to one-dimensional blood flow, Journal of Computational Physics, 300 (2015), 423-437.

[169] J. Neusser, C. Rohde and V. Schleper, Relaxation of the Navier-Stokes-Korteweg equations for compressible two-phase flow with phase transition, International Journal for Numerical Methods in Fluids, 79 (2015), 615-639.

[170] S. Nilsson, N.A. Petersson, B. Sjögreen and H.-O. Kreiss, Stable difference approximations for the elastic wave equation in second order formulation, SIAM Journal on Numerical Analysis, 45 (2007), 1902-1936.

[171] T. Nonomura, N. Iizuka and K. Fujii, Freestream and vortex preservation properties of high-order WENO and WCNS on curvilinear grids, Computers \& Fluids, 39 (2010), $197-214$.

[172] J. Nunez-de la Rosa and C.-D. Munz, XTROEM-FV: a new code for computational astrophysics based on very high order finite-volume methods - I. Magnetohydrodynamics, Monthly Notices of the Royal Astronomical Society, 455 (2016), 3458-3479. 
[173] A.L. Ortega, M. Lombardini, P.T. Barton, D.I. Pullin and D.I. Meiron, RichtmyerMeshkov instability for elastic-plastic solids in converging geometries, Journal of the Mechanics and Physics of Solids, 76 (2015), 291-324.

[174] S. Pang, K. Hirano, R. Fabbro and T. Jiang, Explanation of penetration depth variation during laser welding under variable ambient pressure, Journal of Laser Applications, 27 (2015), UNSP 022007.

[175] K.T. Panourgias and J.A. Ekaterinaris, A discontinuous Galerkin approach for highresolution simulations of three-dimensional flows, Computer Methods in Applied Mechanics and Engineering, 299 (2016), 245-282.

[176] B. Perthame, Second-order Boltzmann schemes for compressible Euler equations in one and two space dimensions, SIAM Journal on Numerical Analysis, 29 (1992), 1-19.

[177] B. Perthame and C.-W. Shu, On positivity preserving finite volume schemes for Euler equations, Numerische Mathematik, 73 (1996), 119-130.

[178] S. Peskin, The immersed boundary method, Acta Numerica, 11 (2002), 1-39.

[179] S. Pirozzoli, Conservative hybrid compact-WENO schemes for shock-turbulence interaction, Journal of Computational Physics, 178 (2002), 81-117.

[180] D. Ponziani, S. Pirozzoli and F. Grasso, Development of optimized weighted-ENO schemes for multiscale compressible flows, International Journal for Numerical Methods in Fluids, 42 (2003), 953-977.

[181] K.G. Pressel, C.M. Kaul, T. Schneider, Z. Tan and S. Mishra, Large-eddy simulation in an anelastic framework with closed water and entropy balances, Journal of Advances in Modeling Earth Systems, 7 (2015), 1425-1456.

[182] T. Qin, C.-W. Shu and Y. Yang, Bound-preserving discontinuous Galerkin methods for relativistic hydrodynamics, Journal of Computational Physics, 315 (2016), 323-347. 
[183] J.-M. Qiu and C.-W. Shu, Positivity preserving semi-Lagrangian discontinuous Galerkin formulation: theoretical analysis and application to the Vlasov-Poisson system, Journal of Computational Physics, 230 (2011), 8386-8409.

[184] J.-X. Qiu and C.-W. Shu, Hermite WENO schemes and their application as limiters for Runge-Kutta discontinuous Galerkin method: one-dimensional case, Journal of Computational Physics, 193 (2004), 115-135.

[185] J.-X. Qiu and C.-W. Shu, Hermite WENO schemes and their application as limiters for Runge-Kutta discontinuous Galerkin method II: two dimensional case, Computers \& Fluids, 34 (2005), 642-663.

[186] J.-X. Qiu and C.-W. Shu, Runge-Kutta discontinuous Galerkin method using WENO limiters, SIAM Journal on Scientific Computing, 26 (2005), 907-929.

[187] J.-X. Qiu and C.-W. Shu, A comparison of troubled-cell indicators for Runge-Kutta discontinuous Galerkin methods using weighted essentially nonoscillatory limiters, SIAM Journal on Scientific Computing, 27 (2005), 995-1013.

[188] W.H. Reed and T.R. Hill, Triangular mesh methods for the neutron transport equation, Los Alamos Scientific Laboratory Report LA-UR-73-479, Los Alamos, NM, 1973.

[189] J.-F. Remacle, J. Flaherty and M. Shephard, An adaptive discontinuous Galerkin technique with an orthogonal basis applied to Rayleigh-Taylor flow instabilities, SIAM Review, 45 (2003), 53-72.

[190] X. Ren and C. Gu, Application of a discontinuous Galerkin method on the compressible flow in the transonic axial compressor, Applied Thermal Engineering, 93 (2016), 707717. 
[191] Y. Ren, M. Liu and H. Zhang, A characteristic-wise hybrid compact-WENO scheme for solving hyperbolic conservation laws, Journal of Computational Physics, 192 (2003), 365-386.

[192] B. Reuter, V. Aizinger and H. Koestler, A multi-platform scaling study for an OpenMP parallelization of a discontinuous Galerkin ocean model, Computer and Fluids, 117 (2015), 325-335.

[193] G. Ribert, D. Taieb and V. Yang, Large-eddy simulation of a supercritical channel flow using a shock capturing numerical scheme, Computer and Fluids, 117 (2015), 103-113.

[194] B. Rivière, Discontinuous Galerkin methods for solving elliptic and parabolic equations. Theory and implementation, SIAM, Philadelphia, 2008.

[195] J.A. Rossmanith and D.C. Seal, A positivity-preserving high-order semi-Lagrangian discontinuous Galerkin scheme for the Vlasov-Poisson equations, Journal of Computational Physics, 230 (2011), 6203-6232.

[196] A. Ruttgers, M. Griebel, L. Pastrik, H. Schmied, D. Wittmann, A. Scherrieble, A. Dinkelmann and T. Stegmaier, Simulation of the oil storage process in the scopa of specialized bees, Computers \& Fluids, 119 (2015), 115-130.

[197] K. Schaal, A. Bauer, P. Chandrashekar, R. Pakmor, C. Klingenberg and V. Springel, Astrophysical hydrodynamics with a high-order discontinuous Galerkin scheme and adaptive mesh refinement, Monthly Notices of the Royal Astronomical Society, 453 (2015), 4278-4300.

[198] F. Schneider, J. Kall and G. Alldredge, A realizability-preserving high-order kinetic scheme using WENO reconstruction for entropy-based moment closures of linear kinetic equations in slab geometry, Kinetic and Related Models, 9 (2016), 193-215. 
[199] K. Sebastian and C.-W. Shu, Multidomain WENO finite difference method with interpolation at subdomain interfaces, Journal of Scientific Computing, 19 (2003), 405-438.

[200] C. Shen, J.M. Qiu and A. Christlieb, Adaptive mesh refinement based on high order finite difference WENO scheme for multi-scale simulations, Journal of Computational Physics, 30 (2011), 3780-3802.

[201] J. Shi, C. Hu and C.-W. Shu, A technique of treating negative weights in WENO schemes, Journal of Computational Physics, 175 (2002), 108-127.

[202] C.-W. Shu, TVB uniformly high-order schemes for conservation laws, Mathematics of Computation, 49 (1987), 105-121.

[203] C.-W. Shu, Total-variation-diminishing time discretizations, SIAM Journal on Scientific and Statistical Computing, 9 (1988), 1073-1084.

[204] C.-W. Shu, Essentially non-oscillatory and weighted essentially non-oscillatory schemes for hyperbolic conservation laws, in Advanced Numerical Approximation of Nonlinear Hyperbolic Equations, B. Cockburn, C. Johnson, C.-W. Shu and E. Tadmor (Editor: A. Quarteroni), Lecture Notes in Mathematics, volume 1697, Springer, Berlin, 1998, pp.325-432.

[205] C.-W. Shu, High order weighted essentially nonoscillatory schemes for convection dominated problems, SIAM Review, 51 (2009), 82-126.

[206] C.-W. Shu, Discontinuous Galerkin methods: general approach and stability, in Numerical Solutions of Partial Differential Equations, S. Bertoluzza, S. Falletta, G. Russo and C.-W. Shu, Advanced Courses in Mathematics CRM Barcelona, Birkhäuser, Basel, 2009, pp.149-201.

[207] C.-W. Shu and S. Osher, Efficient implementation of essentially non-oscillatory shockcapturing schemes, Journal of Computational Physics, 77 (1988), 439-471. 
[208] C.-W. Shu and S. Osher, Efficient implementation of essentially non-oscillatory shockcapturing schemes, II, Journal of Computational Physics, 83 (1989), 32-78.

[209] G.P. Silveira and L.C. de Barros, Analysis of the dengue risk by means of a TakagiSugeno-style model, Fuzzy Sets and Systems, 277 (2015), 122-137.

[210] B. Sjögreen and N.A. Petersson, A Cartesian embedded boundary method for hyperbolic conservation laws, Communications in Computational Physics, 2 (2007), 1199-1219.

[211] B. Sjögreen, H.C. Yee and M. Vinokur, On high order finite-difference metricdiscretizations satisfying GCL on moving and deforming grids, Journal of Computational Physics, 265 (2014), 211-220.

[212] W. Su, A.A. Alexeenko and G. Cai, A parallel Runge-Kutta discontinuous Galerkin solver for rarefied gas flows based on 2D Boltzmann kinetic equations, Computers \& Fluids, 109 (2015), 123-136.

[213] T. Sumi and T. Kurotaki, A new central compact finite difference formula for improving robustness in weighted compact nonlinear schemes, Computers \& Fluids, 123 (2015), $162-182$.

[214] W. Sun, S.H. Won, X. Gou and Y. Ju, Multi-scale modeling of dynamics and ignition to flame transitions of high pressure stratified $n$-heptane/toluene mixtures, Proceedings of the Combustion Institute, 35 (2015), 1049-1056.

[215] N. Tacik, F. Foucart, H.P. Pfeiffer, R. Haas, S. Ossokine, J. Kaplan, C. Muhlberger, M.D. Duez, L.E. Kidder, M.A. Scheel and B. Szilagyi, Binary neutron stars with arbitrary spins in numerical relativity, Physical Review D, 92 (2015), 124012.

[216] S. Tan and C.-W. Shu, Inverse Lax-Wendroff procedure for numerical boundary conditions of conservation laws, Journal of Computational Physics, 229 (2010), 8144-8166. 
[217] S. Tan and C.-W. Shu, A high order moving boundary treatment for compressible inviscid flows, Journal of Computational Physics, 230 (2011), 6023-6036.

[218] S. Tan and C.-W. Shu, Inverse Lax-Wendroff procedure for numerical boundary conditions of hyperbolic equations: survey and new developments, in "Advances in Applied Mathematics, Modeling and Computational Science", R. Melnik and I. Kotsireas, Editors, Fields Institute Communications 66, Springer, New York, 2013, pp.41-63.

[219] S. Tan, C. Wang, C.-W. Shu and J. Ning, Efficient implementation of high order inverse Lax-Wendroff boundary treatment for conservation laws, Journal of Computational Physics, 231 (2012), 2510-2527.

[220] P.D. Thomas and C.K. Lombard, Geometric conservation law and its application to flow computations on moving grids, AIAA Journal, 17 (1979), 1030-1037.

[221] S. Tirupathi, J.S. Hesthaven, Y. Liang and M. Parmentier, Multilevel and local timestepping discontinuous Galerkin methods for magma dynamics, Computational Geosciences, 19 (2015), 965-978.

[222] A. Tiwari, C. Pantano and J.B. Freund, Growth-and-collapse dynamics of small bubble clusters near a wall, Journal of Fluid Mechanics, 775 (2015), 1-23.

[223] N. Tosi, C. Stein,L. Noack, C. Huttig, P. Maierova, H. Samuel, D.R. Davies, C.R. Wilson, S.C. Kramer, C. Thieulot, A. Glerum, M. Fraters, W. Spakman, A. Rozel and P.J. Tackley, A community benchmark for viscoplastic thermal convection in a 2-D square box, Geochemistry Geophysics Geosystems, 16 (2015), 2175-2196.

[224] M.P. Ueckermann and P.F.J. Lermusiaux, Hybridizable discontinuous Galerkin projection methods for Navier-Stokes and Boussinesq equations, Journal of Computational Physics, 306 (2016), 390-421. 
[225] J.J. van der Dussen, S.R. de Roode, S. Dal Gesso and A.P. Siebesma, An LES model study of the influence of the free tropospheric thermodynamic conditions on the stratocumulus response to a climate perturbation, Journal of Advances in Modeling Earth Systems, 7 (2015), 670-691.

[226] B. van Leer, S. Nomura and M. van Raalte, A discontinuous Galerkin method for diffusion based on recovery, AIAA Paper 2007-4083, 2007.

[227] M. van Raalte and B. van Leer, Bilinear forms for the recovery-based discontinuous Galerkin method for diffusion, Communications in Computational Physics, 5 (2009), 683-693.

[228] F. Vilar and C.-W. Shu, Development and stability analysis of the inverse Lax-Wendroff boundary treatment for central compact schemes, ESAIM: Mathematical Modelling and Numerical Analysis $\left(M^{2} A N\right), 49$ (2015), 39-67.

[229] F. Vilar, C.-W. Shu and P.-H. Maire, Positivity-preserving cell-centered Lagrangian schemes for multi-material compressible flows: From first-order to high-orders. Part I: The one-dimensional case, Journal of Computational Physics, 312 (2016), 385-415.

[230] F. Vilar, C.-W. Shu and P.-H. Maire, Positivity-preserving cell-centered Lagrangian schemes for multi-material compressible flows: From first-order to high-orders. Part II: The two-dimensional case, Journal of Computational Physics, 312 (2016), 416-442.

[231] R.M. Visbal and D.V. Gaitonde, On the use of higher-order finite-difference schemes on curvilinear and deforming meshes, Journal of Computational Physics, 181 (2002), $155-185$.

[232] M.J. Vuik and J.K. Ryan, Multiwavelet troubled-cell indicator for discontinuity detection of discontinuous Galerkin schemes, Journal of Computational Physics, 270 (2014), 138-160. 
[233] C. Wang, J. Ding, S. Tan and W. Han, High order numerical simulation of detonation wave propagation through complex obstacles with the inverse Lax-Wendroff treatment, Communications in Computational Physics, 18 (2015), 1264-1281.

[234] C. Wang, X. Dong and C.-W. Shu, Parallel adaptive mesh refinement method based on WENO finite difference scheme for the simulation of multi-dimensional detonation, Journal of Computational Physics, 298 (2015), 161-175.

[235] L. Wang, H. Chen, X. Ye and X. Yao, Study on load characteristics of underwater explosion using RKDG-LS-DGF and BEM, Shock and Vibration, (2015), 165252.

[236] W. Wang, J. Guzmán and C.-W. Shu, The multiscale discontinuous Galerkin method for solving a class of second order elliptic problems with rough coefficients, International Journal of Numerical Analysis and Modeling, 8 (2011), 28-47.

[237] W. Wang, X. Li and C.-W. Shu, The discontinuous Galerkin method for the multiscale modeling of dynamics of crystalline solids, Multiscale Modeling and Simulation, 7 (2008), 294-320.

[238] W. Wang and C.-W. Shu, The WKB local discontinuous Galerkin method for the simulation of Schrödinger equation in a resonant tunneling diode, Journal of Scientific Computing, 40 (2009), 360-374.

[239] W. Wang, C.-W. Shu, H.C. Yee, D.V. Kotov and B. Sjögreen, High order finite difference methods with subcell resolution for stiff multispecies detonation capturing, Communications in Computational Physics, 17 (2015), 317-336.

[240] Z.J. Wang, Spectral (finite) volume method for conservation laws on unstructured grids: basic formulation, Journal of Computational Physics, 178 (2002), 210-251. 
[241] Z.J. Wang and R.F. Chen, Optimized weighted essentially nonoscillatory schemes for linear waves with discontinuity, Journal of Computational Physics, 174 (2001), 381404.

[242] Z.J. Wang and H. Gao, A unifying lifting collocation penalty formulation including the discontinuous Galerkin, spectral volume/difference methods for conservation laws on mixed grids, Journal of Computational Physics, 228 (2009), 8161-8186.

[243] L. Wei, Y. He and X. Zhang, Analysis of an implicit fully discrete local discontinuous Galerkin method for the time-fractional KdV equation, Advances in Applied Mathematics and Mechanics, 7 (2015), 510-527.

[244] K. Wu and H. Tang, High-order accurate physical-constraints-preserving finite difference WENO schemes for special relativistic hydrodynamics, Journal of Computational Physics, 298 (2015), 539-564.

[245] L. Wu and C.-W. Shu, Numerical solution of the viscous surface wave with discontinuous Galerkin method, ESAIM: Mathematical Modelling and Numerical Analysis $\left(M^{2} A N\right), 49$ (2015), 1019-1046.

[246] M. Wurst, M. Kessler and E. Kraemer, A high-order discontinuous Galerkin Chimera method for laminar and turbulent flows, Computers \& Fluids, 121 (2015), 102-113.

[247] Y. Xia, A fully discrete stable discontinuous Galerkin method for the thin film epitaxy problem without slope selection, Journal of Computational Physics, 280 (2015), 248260.

[248] Y. Xing, X. Zhang and C.-W. Shu, Positivity preserving high order well balanced discontinuous Galerkin methods for the shallow water equations, Advances in Water Resources, 33 (2010), 1476-1493. 
[249] T. Xiong, J.-M. Qiu and Z. Xu, A parametrized maximum principle preserving flux limiter for finite difference RK-WENO schemes with applications in incompressible flows, Journal of Computational Physics, 252 (2013), 310-331.

[250] T. Xiong, J.-M. Qiu and Z. Xu, High order maximum-principle-preserving discontinuous Galerkin method for convection-diffusion equations, SIAM Journal on Scientific Computing, 37 (2015), A583-A608.

[251] Y. Xu and C.-W. Shu, Local discontinuous Galerkin methods for two classes of twodimensional nonlinear wave equations, Physica D, 208 (2005), 21-58.

[252] Y. Xu and C.-W. Shu, Local discontinuous Galerkin methods for high-order timedependent partial differential equations, Communications in Computational Physics, 7 (2010), 1-46.

[253] Z. Xu, Parametrized maximum principle preserving flux limiters for high order scheme solving hyperbolic conservation laws: one-dimensional scalar problem, Mathematics of Computation, 83 (2014), 2213-2238.

[254] J. Yan and C.-W. Shu, A local discontinuous Galerkin method for KdV type equations, SIAM Journal on Numerical Analysis, 40 (2002), 769-791.

[255] J. Yan and C.-W. Shu, Local discontinuous Galerkin methods for partial differential equations with higher order derivatives, Journal of Scientific Computing, 17 (2002), $27-47$.

[256] Y. Yang and C.-W. Shu, Analysis of optimal superconvergence of discontinuous Galerkin method for linear hyperbolic equations, SIAM Journal on Numerical Analysis, 50 (2012), 3110-3133. 
[257] Y. Yang and C.-W. Shu, Discontinuous Galerkin method for hyperbolic equations involving $\delta$-singularities: negative-order norm error estimates and applications, $\mathrm{Nu-}$ merische Mathematik, 124, (2013), 753-781.

[258] Y. Yang, M. Wan, Y. Shi, K. Yang and S. Chen, A hybrid scheme for compressible magnetohydrodynamic turbulence, Journal of Computational Physics, 306 (2016), 7391.

[259] Y. Yang, D. Wei and C.-W. Shu, Discontinuous Galerkin method for Krause's consensus models and pressureless Euler equations, Journal of Computational Physics, 252 (2013), 109-127.

[260] C. Yu, D. Wang, Z. He and T. Pahtz, An optimized dispersion-relation-preserving combined compact difference scheme to solve advection equations, Journal of Computational Physics, 300 (2015), 92-115.

[261] R. Yu, X.-S. Bai and V. Bychkov, Fractal flame structure due to the hydrodynamic Darrieus-Landau instability, Physical Review E, 92 (2015), 063028.

[262] L. Yuan and C.-W. Shu, Discontinuous Galerkin method based on non-polynomial approximation spaces, Journal of Computational Physics, 218 (2006), 295-323.

[263] L. Yuan and C.-W. Shu, Discontinuous Galerkin method for a class of elliptic multiscale problems, International Journal for Numerical Methods in Fluids, 56 (2008), 1017-1032.

[264] O. Zanotti and M. Dumbser, A high order special relativistic hydrodynamic and magnetohydrodynamic code with space-time adaptive mesh refinement, Computer Physics Communications, 188 (2015), 110-127.

[265] O. Zanotti, F. Fambri and M. Dumbser, Solving the relativistic magnetohydrodynamics equations with ADER discontinuous Galerkin methods, a posteriori subcell limiting and 
adaptive mesh refinement, Monthly Notices of the Royal Astronomical Society, 452 (2015), 3010-3029.

[266] O. Zanotti, F. Fambri, M. Dumbser and A. Hidalgo, Space-time adaptive ADER discontinuous Galerkin finite element schemes with a posteriori sub-cell finite volume limiting, Computers \& Fluids, 118 (2015), 204-224.

[267] D. Zhang, J. Tan and L. Lv, Investigation on flow and mixing characteristics of supersonic mixing layer induced by forced vibration of cantilever, Acta Astronautica, 117 (2015), 440-449.

[268] F. Zhang, R. Yu and X. Bai, Effect of split fuel injection on heat release and pollutant emissions in partially premixed combustion of PRF70/air/EGR mixtures, Applied Energy, 149 (2015), 283-296.

[269] H. Zhang, M. Reggio, T.Y. Trépanier and R. Camarero, Discrete form of the GCL for moving meshes and its implementation in CFD schemes, Computers \& Fluids, 22 (1993), 9-23.

[270] R. Zhang, M. Zhang and C.-W. Shu, On the order of accuracy and numerical performance of two classes of finite volume WENO schemes, Communications in Computational Physics, 9 (2011), 807-827.

[271] S. Zhang, S. Jiang and C.-W. Shu, Development of nonlinear weighted compact schemes with increasingly higher order accuracy, Journal of Computational Physics, 227 (2008), 7294-7321.

[272] X. Zhang and C.-W. Shu, On maximum-principle-satisfying high order schemes for scalar conservation laws, Journal of Computational Physics, 229 (2010), 3091-3120. 
[273] X. Zhang and C.-W. Shu, On positivity preserving high order discontinuous Galerkin schemes for compressible Euler equations on rectangular meshes, Journal of Computational Physics, 229 (2010), 8918-8934.

[274] X. Zhang and C.-W. Shu, Positivity-preserving high order discontinuous Galerkin schemes for compressible Euler equations with source terms, Journal of Computational Physics, 230 (2011), 1238-1248.

[275] X. Zhang and C.-W. Shu, Maximum-principle-satisfying and positivity-preserving high order schemes for conservation laws: Survey and new developments, Proceedings of the Royal Society A, 467 (2011), 2752-2776.

[276] X. Zhang and C.-W. Shu, A minimum entropy principle of high order schemes for gas dynamics equations, Numerische Mathematik, 121 (2012), 545-563.

[277] X. Zhang and C.-W. Shu, Positivity-preserving high order finite difference WENO schemes for compressible Euler equations, Journal of Computational Physics, 231 (2012), 2245-2258.

[278] X. Zhang and S. Tan, A simple and accurate discontinuous Galerkin scheme for modeling scalar-wave propagation in media with curved interfaces, Geophysics, 80 (2015), T83-T89.

[279] X. Zhang, Y. Xia and C.-W. Shu, Maximum-principle-satisfying and positivitypreserving high order discontinuous Galerkin schemes for conservation laws on triangular meshes, Journal of Scientific Computing, 50 (2012), 29-62.

[280] Y. Zhang, W. Liu and B. Wang, Effects of oblique and transverse injection on the characteristics of jet in supersonic crossflow, Acta Astronautica, 115 (2015), 356-366. 
[281] Y. Zhang, W. Wang, J. Guzmán and C.-W. Shu, Multi-scale discontinuous Galerkin method for solving elliptic problems with curvilinear unidirectional rough coefficients, Journal of Scientific Computing, 61 (2014), 42-60.

[282] Y. Zhang, X. Zhang and C.-W. Shu, Maximum-principle-satisfying second order discontinuous Galerkin schemes for convection-diffusion equations on triangular meshes, Journal of Computational Physics, 234 (2013), 295-316.

[283] Y.-T. Zhang and C.-W. Shu, Third order WENO scheme on three dimensional tetrahedral meshes, Communications in Computational Physics, 5 (2009), 836-848.

[284] X. Zhao, Y. Yang and C.E. Seyler, A positivity-preserving semi-implicit discontinuous Galerkin scheme for solving extended magnetohydrodynamics equations, Journal of Computational Physics, 278 (2014), 400-415.

[285] X. Zhong and C.-W. Shu, A simple weighted essentially nonoscillatory limiter for Runge-Kutta discontinuous Galerkin methods, Journal of Computational Physics, 232 (2012), 397-415.

[286] S. Zhou, L.-T. Cheng, H. Sun, J. Che, J. Dzubiella, B. Li and J.A. McCammon, LS-VISM: A software package for analysis of biomolecular solvation, Journal of Computational Chemistry, 36 (2015), 1047-1059.

[287] T. Zhou, Y. Li and C.-W. Shu, Numerical comparison of WENO finite volume and Runge-Kutta discontinuous Galerkin methods, Journal of Scientific Computing, 16 (2001), 145-171.

[288] J. Zhu and J.-X. Qiu, WENO schemes and their application as limiters for RKDG methods based on trigonometric approximation spaces, Journal of Scientific Computing, 55 (2013), 606-644. 
[289] J. Zhu, J.-X. Qiu, C.-W. Shu and M. Dumbser, Runge-Kutta discontinuous Galerkin method using WENO limiters II: unstructured meshes, Journal of Computational Physics, 227 (2008), 4330-4353.

[290] J. Zhu, X. Zhong, C.-W. Shu and J.-X. Qiu, Runge-Kutta discontinuous Galerkin method using a new type of WENO limiters on unstructured meshes, Journal of Computational Physics, 248 (2013), 200-220.

[291] J. Zhu, X. Zhong, C.-W. Shu and J.-X. Qiu, Runge-Kutta discontinuous Galerkin method with a simple and compact Hermite WENO limiter, Communications in Computational Physics, 19 (2016), 944-969.

[292] J. Zhu, X. Zhong, C.-W. Shu and J.-X. Qiu, Runge-Kutta discontinuous Galerkin method with a simple and compact Hermite WENO limiter on unstructured meshes, submitted to Communications in Computational Physics.

[293] W. Zhu, L.-L. Feng, Y. Xia, C.-W. Shu, Q. Gu and L.-Z. Fang, Turbulence in the intergalactic medium: solenoidal and dilatational motions and the impact of numerical viscosity, The Astrophysical Journal, 777 (2013), 48. 